\title{
Direct numerical simulations of particle sedimentation with heat transfer using the Lattice Boltzmann method
}

Bo Yang ${ }^{1}$, Sheng Chen*1,2,3, Kai Liu ${ }^{1}$

1 State Key Laboratory of Coal Combustion, School of Energy and Power Engineering, Huazhong University of Science and Technology, Wuhan, 430074, China

2 Institute for Modelling and Simulation in Fluodynamics, Nanoscience and Industrial Mathematics "Gregorio Millan Barbany", Universidad Carlos III de Madrid, Leganes 28911, Spain

3 Faculty of Engineering, The University of Nottingham, University Park, Nottingham NG7 2RD, UK

*Corresponding author: Faculty of Engineering, University of Nottingham.

E-mail address: shengchen.hust@gmail.com

\begin{abstract}
In most realistic gas-solid flow, the difference of particles' temperature is significant. The heat transfer induced by temperature difference between particles will influence the behavior of gas-solid flow critically. In order to deepen our insights into this important topic, in this work three typical cases: (1) double hot particles, (2) double cold particles, and (3) one hot and one cold particle, are investigated with the aid of direct numerical simulation of the Lattice Boltzmann method. A comprehensive comparison is carried out between them and some new interesting phenomena are observed. Our results show that thermal convection between particles will influence their behaviors significantly.
\end{abstract}

\section{Introduction}

Particle sedimentation exists in many natural and industrial processes, such as sand deposition in rivers and particle fluidization in fluidized bed reactors. In the past decades, the behaviors of suspended particles settling under gravity have been investigated experimentally and numerically extensively by many researchers [1]-[3]. It has been demonstrated that heat transfer between particles with different temperature has a strong influence on particulate flow performance. Gan et al. [4] 
investigated the effects of heat transfer on the sedimentation of double cold and hot particles. It was found that cold particles would tend to repel each other while hot particles would attract each other. The effects of Grashof numbers (Gr) on the interaction between two cold particles were also uncovered. With increasing in Gr, the interactions of repelling and attracting between particles become more evident. However, many important aspects were omitted in their investigation, such as effects of the walls and high Grashof numbers. Feng et al. [5] studied the sedimentation of 56 heated circular particles in an enclosure. In their work, the particles were cooled gradually due to energy exchange with the fluid. The authors observed that the trailing particles in the thermal wakes of the leading particles had a higher temperature. Zahra Hashemi et al. [6] simulated the sedimentation of 30 hot particles in an enclosure. The collective behavior of these hot particles was investigated. It was found that the average settling velocity of hot particles were much lower than the isothermal case. Recently, Henrik Ström et al. [7] studied the sedimentation of two hot particles with an internal heat source. The authors revealed that when the internal heat sources were large enough, the initial drafting was reversed and no kissing or tumbling occurred. Zhang et al. [8] made a comparison of sedimentation process of 185 and 504 particles among three cases: cold particles, hot particles and isothermal particles. The particle distribution patterns of three cases with isothermal contours during sedimentation were obtained. The cold particles obviously settled more efficiently than others. However, many important flow characteristics, such as vortex and velocity distribution, were not investigated. Zhang et al. [9] also investigated the behaviors of 5000 and 8125 particles with heat transfer, and uncovered the distribution characteristics of particles' velocity and position. Their results revealed that the thermal buoyancy had a great effect on the sedimentation efficiency of the solid particles. When considering heat transfer, the interface between the hot particle aggregated and lower fluid was more unstable. Our previous works [10]-[11] identified three interaction regimes (repulsion, attraction and transition regime) between double settling particles based on initial configurations, and investigated the effects of heat transfer on double cold particles in each regime. Although the sedimentation of thermal particles has been investigated in mentioned literature, the effects of Gr on the sedimentation of two hot particles and two cold particles still remain to be absent in some aspects, and these will be uncovered in detail. Then, 
most above literature studies the effects of heat transfer on the motions of two settling particles with the same temperature, while the temperature of particles is usually different in realistic suspension flow. Thus a comprehensive study on sedimentation of two particles with different temperature (namely one hot particle and one cold particle) with various $\mathrm{Gr}$ is investigated for the first time. Lastly, most of above literature studies the sedimentation of thermal particles individually, a comprehensive comparison of interaction regimes among three cases (two hot, two cold, one hot and one cold particles) at the same condition is absent yet. It will be discussed in this paper for the first time.

\section{Numerical method}

In recent years, the Lattice Boltzmann Method (LBM) [12] has been developed to model particulate flows due to its efficiently treating the complex system of moving boundaries [13]-[14]. The immersed boundary method (IBM) was introduced by Peskin [15] and evolved to kinds of computing strategies. It gains popularity because it is straightforward and easier to apply than other boundary approaches [16]-[17]. The direct-forcing IBM has been applied to solving many complex boundary problems [18]-[23] because it has good stability and hasn't adjusted arbitrary parameters. Here, we adopt the direct-forcing method with sharp interface scheme proposed by Kang et al. [18]-[19].

Specifically, the double-population thermal lattice Boltzmann equations are employed to solve flow and temperature field. The evolution equations for fluid flow and temperature field are formulated as

$$
\begin{aligned}
& f_{i}\left(\mathbf{x}+\mathbf{c}_{i} \Delta t, t+\Delta t\right)-f_{i}(\mathbf{x}, t)=\Omega_{i}(f)+F_{i} \Delta t+R_{i} \Delta t \\
& T_{i}\left(\mathbf{x}+\mathbf{c}_{i} \Delta t, t+\Delta t\right)-T_{i}(\mathbf{x}, t)=-\frac{1}{\tau_{T}}\left[T_{i}(\mathbf{x}, t)-T_{i}^{e q}(\mathbf{x}, t)\right]+Q_{i} \Delta t
\end{aligned}
$$

Where $f_{i}(\mathbf{x}, t), T_{i}(\mathbf{x}, t)$ are flow and temperature distribution function, respectively. $\Delta t$ is the time step, $F_{i}$ and $R_{i}$ are the discrete forcing term for the boundary force, the buoyancy force respectively. $\Omega_{i}(f)$ is the discrete collision operator in multiple-relaxation-times (MRT) models and is formulated as [24-Error! Reference source not found.

$$
\Omega_{i}(f)=-\sum_{j}\left(M^{-1} S M\right)_{j i}\left(f_{j}-f_{j}^{e q}\right)
$$


Where $f_{j}^{e q}$ is the equilibrium distribution function and $S=\operatorname{diag}\left(\tau_{0}, \tau_{1}, \tau_{2}, \ldots, \tau_{i-1}\right)^{-1}$ is a positive diagonal matrix. We set $S=\operatorname{diag}\left(1,0.2,0.1,1,1.2,1,1.2,1 / \tau_{f}, 1 / \tau_{f}\right)$ using D2Q9 model, where $\tau_{f}$ is the momentum relaxation time. The discrete velocity $c_{i}$ is defined as

$$
\mathbf{c}_{i}=\left\{\begin{array}{cc}
(0,0) & i=0 \\
c(\cos [(i-1) \pi / 2], \sin [(i-1) \pi / 2]) & i=1,2,3,4 \\
\sqrt{2} c(\cos [(2 i-1) \pi / 4], \sin [(2 i-1) \pi / 4]) & i=5,6,7,8
\end{array}\right.
$$

Where $c=\Delta x / \Delta t$ is the lattice speed and $\Delta x$ is the lattice grid spacing. The equilibrium distribution functions $f_{i}^{e q}$ and $T_{i}^{e q}$ are defined by

$$
\begin{aligned}
& f_{i}^{e q}=w_{i} \rho\left[1+3 \frac{\mathbf{c}_{i} \cdot u}{c^{2}}+4.5 \frac{\left(\mathbf{c}_{i} \cdot u\right)^{2}}{c^{4}}-1.5 \frac{u^{2}}{c^{2}}\right] \\
& T_{i}^{e q}=\frac{T}{5}\left[1+5 \frac{\mathbf{c}_{i} \cdot u}{2 c_{i}^{2}}\right]
\end{aligned}
$$

Where $w_{0}=\frac{4}{9}, w_{1-4}=\frac{1}{9}$ and $w_{5-8}=\frac{1}{36}$. The transfer matrix $M$ for MRT is given by

$$
M=\left(\begin{array}{ccccccccc}
1 & 1 & 1 & 1 & 1 & 1 & 1 & 1 & 1 \\
-4 & -1 & -1 & -1 & -1 & 2 & 2 & 2 & 2 \\
4 & -2 & -2 & -2 & -2 & 1 & 1 & 1 & 1 \\
0 & 1 & 0 & -1 & 0 & 1 & -1 & -1 & 1 \\
0 & -2 & 0 & 2 & 0 & 1 & -1 & -1 & 1 \\
0 & 0 & 1 & 0 & -1 & 1 & 1 & -1 & -1 \\
0 & 0 & -2 & 0 & 2 & 1 & 1 & -1 & -1 \\
0 & 1 & -1 & 1 & -1 & 0 & 0 & 0 & 0 \\
0 & 0 & 0 & 0 & 0 & 1 & -1 & 1 & -1
\end{array}\right)
$$

The forcing term $F_{i}$ can be given by [26]

$$
F_{i}=M^{-1}\left(I-\frac{1}{2} S\right) M_{j i} \bar{F}_{i}
$$

Where $I$ is the unit matrix. Using Guo-Zheng-Shi model [27], $\bar{F}_{i}$ and $Q_{i}$ are defined by

$$
\overline{F_{i}}=w_{i}\left(1-\frac{1}{2 \tau_{f}}\right)\left[\frac{\mathbf{c}_{i}-\mathbf{u}}{c_{s}^{2}}+\frac{\mathbf{c}_{i} \cdot \mathbf{u}}{c_{s}^{4}} \mathbf{c}_{i}\right] \cdot \mathbf{F}
$$




$$
Q_{i}=w_{i}\left(1-\frac{1}{2 \tau_{T}}\right) Q
$$

Where $\boldsymbol{F}$ and $\mathrm{Q}$ is the force density and temperature term from the immersed boundary, respectively. The macroscopic quantities can be given by

$$
\rho=\sum_{i=0} f_{i}, \rho \mathbf{u}=\sum_{i=0} \mathbf{c}_{i} f_{i}+\frac{\Delta t}{2} \mathbf{F}, T=\sum_{i=0} T_{i}+\frac{\Delta t}{2} Q
$$

Using the Chapman-Enskog analysis, the NS and temperature equations can be recovered from the Lattice Boltzmann equations:

$$
\begin{aligned}
& \frac{\partial \rho}{\partial t}+\nabla \cdot \rho \mathbf{u}=0 \\
& \frac{\partial}{\partial t}(\rho \mathbf{u})+\nabla \cdot(\rho \mathbf{u u})=-\nabla p+\nu \nabla \cdot\left[\rho \nabla \mathbf{u}+(\nabla \mathbf{u})^{T}\right]+\mathbf{F} \\
& \frac{\partial T}{\partial t}+\nabla \cdot(\mathrm{T} \mathbf{u})=\alpha \nabla^{2} T+Q
\end{aligned}
$$

where $\alpha$ is the thermal diffusion coefficient.

Here we adopt the exterior sharp interface scheme [18] to solve the moving boundary, in which the forcing nodes are located inside the solid and closest to the boundary. Since the treatments of flow and temperature field are similar, we only explain the solution for velocity boundary. The force density $\boldsymbol{F}$ is given by [18]

$$
\mathbf{F}(\mathbf{x}, t)=2 \rho(\mathbf{x}, t) \frac{\mathbf{u}^{d}(\mathbf{x}, t)-\mathbf{u}(\mathbf{x}, t)}{\Delta t}
$$

Where $\mathbf{u}(\mathbf{x}, t)$ is the velocity in the forcing node and $\mathbf{u}^{d}(\mathbf{x}, t)$ is obtained using bilinear and linear interpolation

$$
\mathbf{u}^{d}(\mathbf{x}, t)=\frac{1}{\Delta_{x} \Delta_{y}}\left\{\mathbf{u}_{b}-\left[\Delta_{x}\left(1-\Delta_{y}\right) \mathbf{u}_{2}+\left(1-\Delta_{x}\right)\left(1-\Delta_{y}\right) \mathbf{u}_{3}+\left(1-\Delta_{x}\right) \Delta_{y} \mathbf{u}_{4}\right\}\right.
$$

or

$$
\mathbf{u}^{d}(\mathbf{x}, t)=\left\{\begin{array}{cc}
\frac{1}{\Delta} \mathbf{u}_{b}-\frac{1-\Delta}{\Delta} \mathbf{u}_{f} & \text { if } \Delta \geq 0.5 \\
2 \mathbf{u}_{b}-2 \Delta \mathbf{u}_{f}-(1-2 \Delta) \mathbf{u}_{f f} & \text { if } \Delta<0.5
\end{array}\right.
$$

The bilinear and linear interpolations are illustrated by Fig. 1. In Eq. (16) subscript b indicates the node on the boundary closest to the forcing node $\mathbf{x}$, subscripts 2, 3, 4 are outside fluid nodes moved from the forcing node, by one grid in y-direction, $\mathrm{x}$-direction, and both directions. $\Delta_{x}$ and $\Delta_{y}$ are distances between nodes $\mathrm{b}$ and 4 in $\mathrm{x}$-direction, and in y-direction. While one of the nodes $2,3,4$ is located on the solid 
area, linear interpolation is used. In Eq. (17) $f$, ff indicate the outside fluid nodes migrated from the forcing node, by one and two grid in either $\mathrm{x}$ - or $\mathrm{y}$-direction, and $\Delta$ is the distance between nodes $\mathrm{b}$ and $\mathrm{f}$.

Using Boussinesq approximation, the discrete forcing term $R_{i}$ is expressed as [27]

$$
\begin{aligned}
& R_{i}=w_{i}\left[\frac{\mathbf{c}_{i}-\mathbf{u}}{c_{s}^{2}}+\frac{\mathbf{c}_{i} \cdot \mathbf{u}}{c_{s}^{4}} \mathbf{c}_{i}\right] \cdot \mathbf{R} \\
& \mathbf{R}=-\rho_{0} \beta\left(T-T_{0}\right) \mathbf{g}
\end{aligned}
$$

where $\rho_{0}$ and $T_{0}$ are the average density and temperature of fluid, $\mathbf{g}$ is the gravity vector, and $\beta$ is thermal expansion coefficient of fluid and is given by

$$
\beta=\frac{G r \cdot v^{2}}{d^{3} g \Delta T}
$$

Where $d$ is the diameter of particles in this paper, $\Delta T=T_{s}-T_{0}$ is the temperature gradient between particles and fluid and $\mathrm{Gr}$ is the Grashof number.

On the basis of Newtonian law, following equations are solved to obtain the motion of particles.

$$
\begin{aligned}
& M_{i} \frac{d U_{i}(t)}{d t}=F_{i}(t) \\
& I_{i} \cdot \frac{\Omega_{i}(t)}{d t}+\Omega_{i}(t) \times\left[I_{i} \cdot \Omega_{i}(t)\right]=T_{i}(t)
\end{aligned}
$$

Where $M_{i}, I_{i}$ are the mass and inertial tensor of the $i$ th particle, $F_{i}(\mathrm{t})$ and $T_{i}(\mathrm{t})$ are the total force and toque acting on the $i$ th particle, $U_{i}(t)$ and $\Omega_{i}(t)$ are translational and angular velocity of the $i$ th particle, respectively.

Repulsive force is introduced to solve the particle-particle collisions when the gap between two particles exceeds a given threshold [28]:

$$
F_{i j}=\left\{\begin{array}{cl}
0 & \left|x_{i}-x_{j}\right|>R_{i}+R_{j}+\zeta \\
\frac{c_{i j}}{\varepsilon}\left(\frac{\left|x_{i}-x_{j}\right|-R_{i}-R_{j}-\zeta}{\zeta}\right)^{2} \frac{\left(x_{i}-x_{j}\right)}{\left|x_{i}-x_{j}\right|} & \left|x_{i}-x_{j}\right| \leq R_{i}+R_{j}+\zeta
\end{array}\right.
$$

where $x_{i}, x_{j}$ are the center positions of two particles, and $R_{i}, R_{j}$ are the radius of two particles, respectively. $\mathrm{c}_{i j}$ is the force scaling factor for the sedimentation problems; $\varepsilon$ is the stiffness parameter for collisions, which is set to be $d x^{2} ; \zeta$ is the threshold or "safe zone", and if the gap is less than the given threshold $\zeta$, a strong repulsive force $F_{i j}$ is implemented on each particle to keep the particles apart. In this paper, we set $\zeta$ $=2 d x$. Note that in this Force Model, the collision particles can't contact each other 
physically due to the threshold $\zeta$ and so the friction, deformation and viscous lubrication processes are not considered here.

\section{Simulation results and discussion}

In this section, sedimentation of two circular particles in Newtonian fluid is numerically investigated. The investigated domain is illustrated by Fig. 2. As mentioned above, three representative cases are considered: two hot particles (case 1), two cold particles (case 2), and one hot and one cold particle (case 3). The sizes of the particles are identical, with a diameter $d=0.5 \mathrm{~cm}$ and density $\rho_{p}=1.01 \mathrm{~g} / \mathrm{cm}^{3}$. Initially, the density of fluid is set to $\rho_{f}=1.0 \mathrm{~g} / \mathrm{cm}^{3}$ and the non-dimensional temperature of fluid is set to 0 . The gravity constant $\mathrm{g}$ is set to $-981 \mathrm{~g} / \mathrm{cm}^{3}$. The non-dimensional temperature of hot and cold particles is set to 1 and -1 , respectively. The Prandtl number Pr is fixed to 0.7 and the range of Grashof number Gr is 0-2000. The grid spacing $d x$ is set as $d x=0.025 \mathrm{~cm}$, and the time step is set as $d t=0.001042 \mathrm{~s}$. The reference Reynolds number Re is set to 84 , which is defined by

$$
\operatorname{Re}=\frac{U_{c} d}{v}, U_{c}=\sqrt{\pi d\left(\frac{\rho_{p}}{\rho_{f}}-1\right) g / 2}
$$

where $v$ is the fluid viscosity. In LB the pressure of flow field can be obtained by $p=\rho \cdot c_{s}^{2}$, where $\rho$ is the density and $c_{s}=c / \sqrt{3}$ is the Lattice sound speed. The two particles begin to accelerate in rest fluid at $\mathrm{t}=0$ due to the gravity force. Here we only consider the configurations: $L / d=2.0, \theta=90^{\circ}$. The boundary conditions are given as

Top and bottom boundary: $\partial T / \partial y=0, \partial u / \partial y=0 ;$ left and right boundary: $T=0, u=0$.

Our numerical code has been validated in our previous work [11], so we do not repeat it here for clarity.

\section{1 sedimentation of two hot particles}

In this section, the sedimentation of two hot particles under various $\mathrm{Gr}$ is investigated. By comparing the results at different $\mathrm{Gr}$, the effects of $\mathrm{Gr}$ on the behaviors of hot particles are uncovered.

At low Gr (e.g. Gr=0, 500, 1000), the two hot particles will experience a DKT 
process. The DKT and separating stage of hot particles rely closely on the Gr, as illustrated in Fig. 3. The separating stage indicates the process in which the two particles separate from each other after the DKT. Here and hereafter, the time is normalized by $t^{*}=\frac{t U_{c}}{d}$. With the increasing of Gr, the startup of DKT is postponed and its period is prolonged. During the separating stage, the repulsion force between the two hot particles becomes weak.

Fig. 4 depicts the horizontal and vertical velocity of two hot particles with various Gr. In the present work we take $\mathrm{Gr}=500$ as a representative to discuss it (see Fig. 4(b)). In the beginning settling, particles' vertical velocity increases almost linearly with time until the trailing particle's (particle 1) velocity exceeds the leading one (particle 2) (namely drafting process). When the two hot particles become to contact with each other, the trailing particle's velocity decreases sharply to that of the leading particle's due to the short-range particle-particle force. Then during the tumbling process the two hot particles have the same vertical velocity and the velocity maintains nearly constant. In succession, during the separating stage $\left(84.3<\mathrm{t}^{*}<100\right)$, firstly the two hot particles experience strong drag force and their settling velocities decrease linearly, and then the leading particle's velocity accelerates to approaching that of the trailing one. After the separating stage, their settling velocities decrease to about 2.0 and reach a relatively steady state.

The effects of Gr on the settling velocities of the two hot particles are also significant. Against Gr increases, the tumbling velocities of two hot particles decrease. After separating stage, at $\mathrm{Gr}=0$ settling velocities maintain about 2.34, while at $\mathrm{Gr}>0$ the settling velocities decrease slowly with periodic oscillations. It will be explained below.

It is observed that the variations of horizontal velocities of the two hot particles depend closely on Gr. As shown in Fig. 4(b), when $t^{*}<74.6$ the horizontal settling velocities maintain zero. During $74.6<\mathrm{t}^{*}<84.3$ (during the tumbling process) two hot particles migrate to same side, and theirs horizontal velocities increase to maximum value and then decrease sharply to zero with fluctuations at the same frequency. As pointed out by Gan et al. [4], vortex shedding causes the oscillations of horizontal velocities of the settling particles. Moreover, with the increasing Gr, the horizontal velocities of two hot particles fluctuates slightly milder. It indicates that heat convection between hot particles and surrounding fluid resists the vortex shedding. 
The Gr also influences the variations of hot particles' angular velocities. Fig. 5 depicts the variations of angular velocities of the two hot particles with various Gr. As shown in Fig. 5(b) (Gr=500), when $t^{*}<48$ angular velocities of the two hot particles maintain about zero. During $48<\mathrm{t}^{*}<85.6$ (during the tumbling process) they increase fast up to a maximum value in the opposite directions. In succession, during $85.6<\mathrm{t}^{*}<101.8$ (during the separating process) they decrease fast down to about zero. Lastly, they fluctuate strongly with high frequencies around zero. With high Gr, angular velocities of the two hot particles fluctuate with a bit larger amplitudes.

Fig. 6 plots distributions of vertical velocity and vorticity magnitude contours with various $\mathrm{Gr}$ during the tumbling stage. As pointed out by $\mathrm{Hu}$ et al.[29], the two particles would form a "long body" because their wakes would merge. With Gr increasing, as expected, the moving fluid beside the long body has higher upward velocity, but fluid in the wake of the long body has lower downward velocity. These results can be explained by the fact that the upward stream induced by heat convection between hot particles and surrounding fluid influences strongly the moving fluid. In addition, against Gr increasing, the length of connected-wake is reduced.

As Gr $>1000$, the behavior of sedimentation becomes more complicated. As illustrated by Fig. 7(a), two hot particles at $\mathrm{Gr}=1500$ experience not only a DKT process but also an extra drafting process, in comparison with its low Gr counterpart. By measuring the time duration of tumbling, as expected, the tumbling process begins later and persists longer than its low Gr counterpart. In addition, we observe a small increment of distance between the two particles during $0<\mathrm{t}^{*}<10$. As shown by Fig. $7(\mathrm{c})$, during the extra drafting process $\left(121<\mathrm{t}^{*}<250\right)$ one can observe that both the horizontal and vertical distance between two particles decrease quickly. It can also be reflected by the history of horizontal and vertical velocities illustrated by Fig. 7(b), in which the area between two velocity curves indicates relative displacement. The leading particle always moves a little faster than the trailing particle in horizontal direction. It, together with the vertical velocity difference between the two hot particles, causes the extra drafting process. In addition, as expected, at $\mathrm{Gr}=1500$ the settling velocities experience more significant periodic oscillations since $t^{*}>300$ compared to theirs low Gr counterpart.

When Gr gets up to 2000, completely different process of two hot particles is 
observed, as shown by Fig. 8. The curve of the relative distance between two hot particles looks like a parabola before $\mathrm{t}^{*}=220$ and there is no DKT process. Thereafter, the distance increases relatively slowly with fluctuations during $220<\mathrm{t}^{*}<396$, and then it begins to decrease. For the horizontal direction, settling velocities fluctuate, especially for the trailing particle's. Along the vertical direction, settling velocity of the leading particle increases sharply to about 2 and then decreases slowly, and since $t^{*}>200$ it experiences a periodic fluctuation. For the trailing particle, it will be vertically accelerated to about 1.8 and in succession decelerates sharply to 0 . Then it will be accelerated again to about 0.87 in the reversed direction. In succession, similar processes are reproduced, i.e., the trailing particle decelerates to 0 and then accelerates in reversed direction once again. As the trailing particle's vertical velocity approaches the leading's, it fluctuates substantially: sometime it will move faster than the leading one while the rest time it is slower than the leading one.

As mentioned above, the time evolution of velocities and distance of two hot particles at $\mathrm{Gr}=2000$ is extraordinary, we will try to reveal the reasons. As clearly shown in Fig. 9(a, b), the hot upward stream induced by the leading particle impacts strongly the movement of the trailing particle. This impacting force reduces the vertical velocity of the trailing particle during $17<t^{*}<194$ (see Fig. 8(c)). As pointed out by Fortes et al. [30], the sedimentation of two particles aligned vertical in a Newtonian fluid was dynamically unstable to small disturbances and the asymmetric wake induced by the small axial perturbations would destabilize the couple. As illustrated in Gan et al. [4], the upward stream due to natural convection is unstable. If the leading particle deviates slightly from the channel centre due to small disturbances, the stream starts to oscillate and obtains a serpentine shape. Consequently local pressure difference in the hot wake leads to the strong oscillations of the trailing particle in the horizontal direction, and thus results in the trailing particle shifting from the channel centre to right side (see Fig. 8(b), Fig. 9(c)). Since during $194<t^{*}<250$ the force exerted by upward stream becomes weak, the trailing particle accelerates downward again. Although the trailing particle migrates to the right side, the flow field around the trailing particle is still affected by the upward stream caused by the leading particle. Fig. 9(e, f) depict the flow characteristics at $t^{*}=289.25$. It is observed that upward stream pushes away the surrounding cold fluid and thus the velocity field the trailing particle passing through is not quite uniform. 
This may lead to the vertical velocity oscillations of the trailing particle with big amplitude.

As shown in Fig. 9(f), upward stream due to the strong heat transfer and external downward flow from particle sedimentation influence the motion of the leading particle. In other words, temperature-induced buoyancy effect, as well as buoyancy force and gravity force control the motion of settling particles. If upward forces including temperature-induced buoyancy effect and buoyancy force exceed the downward gravity, the leading particle slows down (see Fig. 8(c)). The periodic oscillations occur, perhaps because of oscillations of the upward stream (see Fig. 9(f)).

In summary, the heat transfer affects the interaction between two hot particles mainly via vortex shedding and upward stream. For low Gr (0-1000), with increasing Gr, vortex shedding is suppressed and upward stream becomes vigorous. Consequently DKT happens latter and persists longer, and both horizontal and vertical distance between two hot particles decrease. Their settling velocities slows down due to strong buoyancy effects. For high Gr (e.g. 2000), DKT disappears. Hot upward stream is unstable due to strong natural convection. Thus oscillations of hot upward stream induced by the leading particle leads to strong oscillations of the trailing particle both in horizontal and vertical direction.

\section{2 sedimentation of two cold particles}

In this section, the effects of Gr on the behaviors of two cold particles settling in infinite channel are investigated. Although this problem has been studied by Gan et al. [4], many important aspects were omitted in their investigation, such as effects of the walls and variation of Grashof numbers. These effects will be analyzed comprehensively in this paper for the first time.

Fig. 10(a) gives the distance between two cold particles as a function of time under various Gr. As expected, cold particles experience shorter tumbling stage and much stronger repulsion force than their isothermal counterparts (namely $\mathrm{Gr}=0$ here). It should be stressed that at $\mathrm{Gr}=1000$ the distance between two cold particles is much bigger than that in the other scenarios. For $\mathrm{Gr} \leq 1000$, the oscillations of horizontal and vertical velocities of two cold particles become stronger with increasing Gr. We take the case of Gr=1000 for example. Fig. 10(b) plots the velocity varies of the two 
cold particles at $\mathrm{Gr}=1000$. For $\mathrm{t}^{*}>150$ the horizontal velocities of both particles experience similar and big oscillations around zero. Compared to their isothermal counterparts (see Fig. 4(a)) in the vertical direction, the tumbling velocities are bigger and for $t^{*}>150$ the velocities oscillate more strongly. In addition, the tumbling velocities keep increasing until the separating stage.

Fig. 10(c) depicts the horizontal and vertical distance between the two cold particles at $\mathrm{Gr}=1000$. It can be observed, after DKT process the horizontal distance increases sharply up to about $25 \mathrm{~d}$ and then oscillates around $25 \mathrm{~d}$. In the vertical direction the distance decreases sharply due to drafting and then maintains constant during the tumbling stage. In succession, the distance increases sharply during the separating stage, and finally keeps about 4d-5d. Combining with Fig. 10(b), it should be noted that strong horizontal velocity oscillations mainly result from repulsion process.

As illustrated by Fig. 11(a), during the tumbling process, the two cold particles are impacted by the downward stream. The connected-wake becomes long (see Fig. 11(b)), and it causes a upward stream encircling the downward stream at the rear of the trailing particle. After separating stage, the stream oscillations and vortex shedding behind cold particles lead to their velocities' oscillations, as shown in Fig. 11(c, d).

As $1500 \leq \mathrm{Gr} \leq 2000$, both horizontal and vertical velocities experience more significant oscillations. Here the case of $\mathrm{Gr}=2000$ is taken as a representative. As shown in Fig. 12(a), the horizontal velocities of the two cold particles experience nearly synchronous and big-amplitude oscillations after separating stage. For the vertical direction shown in Fig. 12(b), during tumbling stage the two cold particles' velocities firstly increase sharply and then decrease quickly, and afterwards fluctuates with gradually decreasing amplitude. After separating stage the vertical velocities also experience oscillations. Fig. 13(a, b) show the distributions of vertical velocity, vorticity magnitude of fluid field at $\mathrm{t}^{*}=34.71$ during tumbling period. From Fig. 13(b), it is clearly observed that the long connected-wake breaks up. It may cause the big-amplitude oscillations of vertical velocities of the two cold particles in the tumbling process. After separating stage the two cold particles interact with each other relatively weakly, and thus they settle in a similar way to one cold particle. Here we take the instant $t^{*}=208.26$ as a representative to explain it. As shown in Fig. 
13(c, d), the distributions of vertical velocity and vorticity magnitude around the two cold particles are relatively independent. As illustrated in Fig. 13(c), the downward flows in the rear of the two cold particles oscillate and form a serpentine shape. Consequently, the vertical velocities of the two cold particles experience strong oscillations (see Fig. 12(b)). As expected, strong vortex shedding (see Fig. 13(d)) leads to oscillations of horizontal velocities of the two cold particles.

As discussed above, the influences of $\mathrm{Gr}$ on the interactions between two cold particles are significant. With increasing Gr, the DKT happens earlier and persists shorter generally, and the oscillations of horizontal and vertical velocities of two cold particles strengthen. After separating stage, downward streams' oscillations in the rear of the two cold particles mainly contribute to oscillations of vertical velocities of two cold particles. For $\mathrm{Gr} \geq 1500$, long connected-wake breaking up perhaps generates the big-amplitude oscillations of vertical velocities of the two cold particles in the tumbling process.

\section{3 sedimentation of one hot and one cold particle}

In this section, the behaviors of one hot and one cold particle settling in Newtonian fluid with various Gr are investigated for the first time. Here, we set the hot particle above the cold particle initially.

As shown in Fig. 14, the tumbling duration of two particles increases with increasing Gr generally. The DKT with various Gr happens nearly at the same instant, but at $\mathrm{Gr}>0$ it persists longer. At Gr=500, after separating stage the distance between the two particles is smaller than that at $\mathrm{Gr}=0$. While for high $\mathrm{Gr}$ (namely 1000, 1500), the distance between the two particles increases violently after separating stage compared to its low Gr counterpart. Through the present study, it is revealed for the first time that there is a critical Gr for the double sedimentation particles.

Fig. 15 gives the time evolution of velocities of the two particles at $\mathrm{Gr}=500,1000$, 1500. The horizontal velocity of the hot particle experiences small oscillations, while the horizontal velocity of the cold particle undergoes strong and periodic oscillations. On the contrary to the sedimentation of two hot particles, with Gr increasing, after separating stage the horizontal velocities of the two particles oscillate strongly, particularly for the leading cold particle. For the vertical velocity, with Gr increasing, the tumbling velocity descends. As shown by Fig. 15(b), we take the case of $\mathrm{Gr}=1000$ 
to illustrate the variation of the vertical velocity. After separating stage the vertical velocity of the cold particle increases to a high value, and then experiences a deceleration process during which the velocity is reduced to about 2.0 , and finally undergoes small-amplitude oscillations. For the hot particle, after separating stage its settling velocity decreases slowly due to heat-induced effects. This can explain the phenomenon that for $\mathrm{Gr}=1000,1500$ the distance between the two particles increases sharply after separating stage.

When $\mathrm{Gr}=2000$, a different phenomenon happens. It is observed in Fig. 16(a) that as the distance between the two particles increases to about 50d, it starts to decrease. As shown in Fig. 16(b), their horizontal velocities oscillate strongly, particularly for the hot particle's. For the vertical direction, during tumbling process, their velocities firstly increase, then decrease sharply and in succession oscillate with small amplitude. After tumbling stage, the cold particle firstly decelerates and then accelerates, and finally its settling velocity experiences small oscillations. While for the hot particle's vertical velocity, after tumbling stage, it fluctuates and since $t^{*}>250$ it experiences strong abnormal oscillations. The hot particle's behavior is similar to that of the trailing particle of two hot particles' sedimentation at $\mathrm{Gr}=2000$. Fig. 17(a, b) plots distributions of vertical velocity and vorticity magnitude of fluid field at $\mathrm{t}^{*}=46.28$ during the tumbling process. Stream above the long body is fragmentary, which is believed to lead to the oscillations of tumbling velocity.

After tumbling stage the leading cold particle's horizontal velocity experiences oscillations at high frequency due to vortex shedding, as shown in Fig. 17(d, f). As illustrated in Fig. 17(c), the stream above the cold particle oscillates, which leads to the oscillations of the cold particle's vertical velocity. Stream oscillations together with the shedding vortex leads to many upward flow regions. When the trailing hot particle passes through these regions, it decelerates. Thus the hot particle experiences repeatedly accelerating and decelerating. Since $t^{*}>250$ vortex shedding happens, shown in Fig. 17(f-2). Consequently, the horizontal velocity of the hot particle also oscillates more strongly.

In summary, as the hot particle is influenced by the cold wake from the cold particle, completely different phenomenons appear. For $\mathrm{Gr}<1000$, the interactions between the hot particle and the cold particle behave like these for two hot particles, such as extended tumbling process and suppressed repulsion process. For Gr $\geq 1000$, 
the natural convection strengthens, causing the obvious velocity distinction between the hot and cold particle and thus sharply increasing distance between them both in horizontal and vertical direction. For $\mathrm{Gr} \geq 2000$, vortex shedding and stream oscillations behind the cold particle occur significantly, leading to strong oscillations of the cold particle's velocity. This also leads to the hot particle's abnormal oscillations.

\subsection{Comparison between three cases}

As discussed above, the behaviors of two settling particles in the three cases have obvious distinctions. Case 1, 2, 3 indicate sedimentation of two hot, two cold, one hot and one cold particle respectively. In addition, we add case 0 to represent the case of two isothermal particles (namely $\mathrm{Gr}=0$ ).

When $\mathrm{Gr}<1000$, repulsion process becomes weak in case 1 and 3, and becomes strong in case 2, as shown in Fig. 18(a). Comparing case 1 with case 3, they are similar except that suppressed effect on repulsion process in case 1 is stronger than in case 3. Fig. 18(b) gives variations of settling velocities in case 2 at $\mathrm{Gr}=500$. During tumbling process the settling velocities increase slowly, and meantime the leading particle's velocity undergoes fluctuations. Referring to Fig. 4(b) and Fig. 15(a), this result is very different from that for two other cases. In addition, one can observe that after separating stage, horizontal velocity oscillations for cold particles are much stronger than for hot particles. These differences among the three cases are a result of thermal effects on vortex shedding behind settling particles.

When $\mathrm{Gr} \geq 1000$, the differences among the three cases are more significant than theirs low Gr counterpart. We take $\mathrm{Gr}=1000$ as a representative to explain it. Fig. 19(a) depicts the comparison of dimensionless distance of two particles among four cases. DKT between the two cold particles happens earliest and persists shortest, while for the two hot particles it happens latest and persists longest. We can express this as $t_{2}^{*}<t_{0}^{*}<t_{3}^{*}<t_{1}^{*}, \Delta t_{2}^{*}<\Delta t_{0}^{*}<\Delta t_{3}^{*}<\Delta t_{1}^{*}$, where $t^{*}, \Delta t^{*}$ indicate the beginning instant and persisting duration of DKT respectively. After separating stage, the distance for the case 1 increases relatively slowly due to small velocity distinction, while the distance for the case 3 increases relatively fast due to large velocity distinction. The distance for the case 2 experiences obvious oscillations around $25 \mathrm{~d}$. Fig. 19(b) plots trajectories of the two particles in the four cases. Particles in case 1 
settle slowest and particles in case 2 settle fastest. Meanwhile particles in case 3 settle in the almost same way with the case 0 . For the horizontal distance between the two particles, the distance for the case 2 is maximum and the distance for the case 1 is minimum.

Fig. 20(a-f) depict vertical velocity and vorticity magnitude contours of fluid field for the three cases at $t^{*}=57.85$. As illustrated by Fig. $20(\mathrm{~b}, \mathrm{e})$, the wake of two cold particles becomes very long and it encircles a large region, leading to a large region of upward stream. The wake of two hot particles is short and thus the upward stream region narrows, as shown in Fig. 20(a, d). These for the case 3 are exactly in the moderate level. Since the tumbling velocity satisfies $U_{t}^{1}<U_{t}^{3}<U_{t}^{2}$ in which superscripts indicate case numbers (refer to Fig. 4(c), Fig. 10(b) and Fig. 15(b)), the velocity of downward fluid in the rear of the two particles also matches the law. As shown in Fig. 20(b, e), it is noted that the maximum velocity region for the case 2 is located in the upside of the downward stream region.

When the interactions between the two particles achieve the relative state $\left(t^{*}>150\right)$, the vortexes and stream behind them for the three cases are also different. Fig. $20(\mathrm{~g}-\mathrm{m})$ plot the flow feature for three cases at $\mathrm{t}^{*}=231.4$. For case 1 in Fig. $20(\mathrm{~g}, \mathrm{j})$, the upward streams and vortexes behind the two hot particles are relatively stable. Thus the oscillations of two hot particles' horizontal and vertical velocities are mild (see Fig. 4(c)). For case 2 in Fig. 20(h, k), the streams behind two cold particles are unstable and oscillate strongly, and strong vortex shedding even results in forming of upward region. Consequently strong oscillations of two cold particles take place in both direction (see Fig. 10(b)). For case 3 in Fig. 20(i, m), the stream and vortex behind the hot particle is similar to that in the case 1 . While for the cold particle, the vortex shedding happens relatively mildly. Thus the oscillations of the cold particle are milder than that in the case 2 .

When Gr gets up to 1500 and above, completely different phenomenons occur among the three cases. We take the $\mathrm{Gr}=2000$ as a representative to explain it in detail. For two hot particles at Gr=2000 (refer to Fig. 8(a)), DKT process disappears. As upward stream induced by the leading particle impacts the trailing particle, drafting process never happens and the trailing particle even moves upwardly for a period. For two cold particles at $\mathrm{Gr}=2000$ (refer to Fig. 12), DKT happens. After separating stage, they settle in a similar way to a cold particle and theirs settling velocities 
experience oscillations due to unstable stream behind them. For the one hot and one cold particle at $\mathrm{Gr}=2000$ (refer to Fig. 16), DKT also happens. During tumbling process, like somewhat the case of two cold particles, their settling velocities also experience oscillations at almost constant amplitude. While after separating stage, like somewhat the case of two hot particles, the trailing hot particle experiences abnormal oscillations in both direction. Meantime, distance between them always increases due to their settling velocity distinction.

In summary, differences of interactions between two particles among the three cases are significant. For low Gr (e.g. 500), the differences among the three cases are a result of thermal effects on vortex shedding behind settling particles. The interactions between the hot particle and the cold particle seem like these between two hot particles, but heat effects for the former are little smaller than for the latter. For middle Gr (e.g. 1000), after separating stage cold particles settle with oscillations and hot particles decelerate slowly with periodic oscillations. Obvious velocity difference is observed between the hot and the cold particle. For high Gr (e.g. 2000), vortex shedding and stream are vigorous. For two hot particles, DKT disappears. For the other two cases, DKT will happen.

\section{Conclusion}

The heat transfer induced by temperature difference between particles will influence the behavior of gas-solid flow significantly. In this work three cases are investigated: (1) sedimentation of two hot particles, (2) sedimentation of two cold particles and (3) sedimentation of one hot particle and one cold particle. Through our analysis, we can draw the following conclusions:

1) The Gr affects the interaction between two hot particles mainly via vortex shedding and upward stream. For low Gr, DKT happens latter and persists longer, and both horizontal and vertical distance between two hot particles decrease. Their settling velocities slows down due to strong buoyancy effects. At $\mathrm{Gr}=1500$, two hot particles experience not only a DKT process but also an extra drafting process. Especially for Gr=2000, DKT disappears. Moreover, oscillations of unstable upward stream induced by the leading particle leads to abnormal oscillations of the trailing particle both in horizontal and vertical direction.

2) For the case of two cold particles, with increasing Gr, DKT happens earlier and 
persists shorter generally, and the oscillations of horizontal and vertical velocities of the two cold particles become strong. Repulsion process between them becomes strongest at about Gr=1000. At high Gr (e.g. Gr=2000), their tumbling velocities experience sharply reducing process with oscillations.

3) For the case of one hot and one cold particle, for $\mathrm{Gr}<1000$, extended tumbling process and suppressed repulsion process are observed. For $1000 \leq \mathrm{Gr}<1500$, the obvious velocity distinction between the hot and cold particle and thus sharply increasing distance between them in both horizontal and vertical direction are observed. For $\mathrm{Gr} \geq 1500$, the two particles' velocities experience abnormal oscillations.

4) Differences of interaction mechanism between two particles among the three cases are significant. For low Gr (e.g. 500), the interaction mechanism between the hot particle and the cold particle seems like these between two hot particles, but heat effects for the former are little smaller than for the latter. For middle Gr (e.g. 1000), after separating stage cold particles' velocities experience strong oscillations and hot particles' velocities decrease slowly with periodic oscillations. For high Gr (e.g. 2000), DKT disappears in the case of two hot particles but it appears in the two other cases.

\section{Acknowledgments}

This work has received funding from the Universidad Carlos III de Madrid, the European Union's Seventh Framework Programme for research, technological development and demonstration under grant agreement No. 600371, el Ministerio de Economía y Competitividad (COFUND2014-51509), el Ministerio de Educación, cultura y Deporte (CEI-15-17) and Banco Santander. We also acknowledge the support from the British Newton Alumni Fellowship Scheme, the National Natural Science Foundation of China (Grant No. 51176061).

\section{Reference}

[1]K.O.L.F. Jayaweera, B.J. Mason, G.W. Slack, The behaviour of clusters of spheres falling in a viscous fluid Part 1. Experiment, J. Fluid Mech. 20 (1964) 121-128.

[2]D.D. Joseph, Y.J. Liu, M. Poletto, J. Feng, Aggregation and dispersion of spheres falling in viscoelastic liquids, J. Non Newtonian Fluid Mech. 54 (1994) 45-86.

[3]J. Wu, R. Manasseh, Dynamics of dual-particles settling under gravity, Int. J. Multiphase Flow 24 (1998) 1343-1358.

[4]Gan H, Chang J, Feng J J, Direct numerical simulation of the sedimentation of solid particles 
with thermal convection, Journal of Fluid Mechanics 481 (2003) 385-411.

[5]Zhi-Gang Feng, Efstathios E. Michaelides, Heat transfer in particulate flows with Direct Numerical Simulation (DNS), International Journal of Heat and Mass Transfer 52 (2009) 777-786.

[6]Zahra Hashemi, Omid Abouali, Reza Kamali, Three dimensional thermal Lattice Boltzmann simulation of heating/cooling spheres falling in a Newtonian liquid, International Journal of Thermal Sciences 82 (2014) 23-33.

[7]Henrik Ström, Srdjan Sasic, Detailed simulations of the effect of particle deformation and particle-fluid heat transfer on particle-particle interactions in liquids, Procedia Engineering 102 (2015) 1563-1572.

[8]Hao Zhang, Aibing Yu, Wenqi Zhong, Yuanqiang Tan, A combined TLBM-IBM-DEM scheme for simulating isothermal particulate flow in fluid, International Journal of Heat and Mass Transfer 91 (2015) 178-189.

[9]Hao Zhang, Haizhuan Yuan, et al., Particulate immersed boundary method for complex fluid-particle interaction problems with heat transfer, Computers and Mathematics with Applications 71 (2016) 391-407

[10] Chuansheng Cao, Sheng Chen, et al., Simulating the interactions of two freely settling spherical particles in Newtonian fluid using lattice-Boltzmann method, Applied Mathematics and Computation 250 (2015) 533-551.

[11] Bo Yang, Sheng Chen, Chuansheng Cao, Zhaohui Liu, Chuguang Zheng, Lattice Boltzmann simulation of two cold particles settling in Newtonian fluid with thermal convection, International Journal of Heat and Mass Transfer 93 (2016) 477-490.

[12] Y.H. Qian, Dominique d'Humi è res, Pierre Lallemand, Lattice BGK models for Navier-Stokes equation, in: EPL (Europhys. Lett.) 17 (6) (1992) 479.

[13] A.J.C. Ladd, Sedimentation of homogeneous suspensions of non-Brownian spheres, Phys. Fluids 9 (1997) 491-499.

[14] C.K. Aidun, L. Yannan, E.J. Ding, Direct analysis of particulate suspensions with inertia using the discrete Boltzmann equation, J. Fluid Mech. 373 (1998) 287-311.

[15] Peskin CS, Flow patterns around heart valves: a numerical method, Journal of Computational Physics 10 (1972) 251-271.

[16] Z.-G. Feng, E.E. Michaelides, The immersed boundary-lattice Boltzmann method for solving fluid - particles interaction problems, J. Comput. Phys. 195 (2) (2004) 602-628.

[17] Z.-G. Feng, E.E. Michaelides, Proteus: a direct forcing method in the simulations of particulate flows, J. Comput. Phys. 202 (1) (2005) 20-51.

[18] Shin K. Kang, Yassin A. Hassan, A comparative study of direct-forcing immersed boundary-lattice Boltzmann methods for stationary complex boundaries, Int. J. Numer. Meth. Fluids 66 (2011) 1132-1158.

[19] Kang S K, Hassan Y A, A direct-forcing immersed boundary method for the thermal lattice 
Boltzmann method, Computers \& Fluids 49 (2011) 36-45.

[20] Delouei, A. A., Nazari, M., Kayhani, M. H., \& Succi, S., Non-Newtonian unconfined flow and heat transfer over a heated cylinder using the direct-forcing immersed boundary - thermal lattice Boltzmann method, Physical Review E, 89 (5) (2014) 053312.

[21] Delouei, A. A., Nazari, M., Kayhani, M. H., \& Succi, S., Immersed boundary - thermal lattice Boltzmann methods for non-Newtonian flows over a heated cylinder: a comparative study. Communications in Computational Physics, 18 (02) (2015) 489-515.

[22] Delouei, A. Amiri, M. Nazari, M. H. Kayhani, S. K. Kang, and S. Succi., Non-Newtonian particulate flow simulation: A direct-forcing immersed boundary - lattice Boltzmann approach, Physica A: Statistical Mechanics and its Applications, 447 (2016) 1-20.

[23] Delouei, A. Amiri, M. Nazari, M. H. Kayhani, and G. Ahmadi., A non-Newtonian direct numerical study for stationary and moving objects with various shapes: An immersed boundary - Lattice Boltzmann approach, Journal of Aerosol Science, 93 (2016) 45-62.

[24] P. Lallemand, L.S. Luo, Theory of the lattice Boltzmann method: dispersion, dissipation, isotropy, Galilean invariance, and stability, Phys. Rev. E 61 (2000) 6546-6562.

[25] F. Higuera, S. Succi, R. Benzi, Lattice gas dynamics with enhanced collisions, Europhys. Lett. 9 (1989) 345.

[26] Zhaoli Guo, Chuguang Zheng, Analysis of lattice Boltzmann equation for microscale gas flows: Relaxation times, boundary, conditions and the Knudsen layer, International Journal of Computational Fluid Dynamics 22 (7) (2008) 465-473.

[27] Guo ZL, Zheng CG, Shi BC, Discrete lattice effects on the forcing term in the lattice Boltzmann method, Phys Rev E 65 (4) (2002):046308.

[28] R. Glowinski, T.W. Pan, T.I. Hesla, D.D. Joseph, J. Périaux, A fictitious domain approach to the direct numerical simulation of incompressible viscous flow past moving rigid bodies: application to particulate flow, J. Comput. Phys. 169 (2001) 363-426.

[29] Howard H. Hu, Daniel D. Joseph, Experiments and direct simulations of fluid particle motions, Video J . Engng Res. 2 (1992) 17-24.

[30] A.F. Fortes, D.D. Joseph, T.S. Lundgren, Nonlinear mechanics of fluidization of beds of spherical particles, J. Fluid Mech. 177 (1987) 467-483. 


\section{Figures}

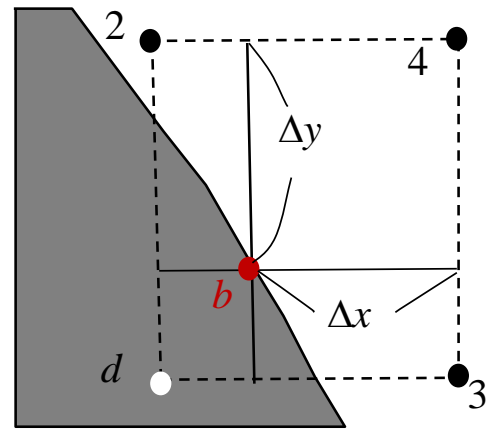

(a)

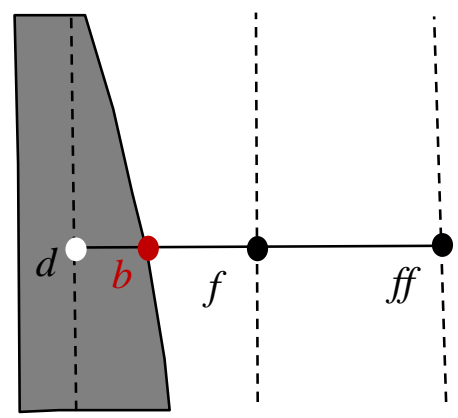

(b)

Fig. 1 Schematic figure of the bilinear and linear interpolations for exterior sharp interface scheme: (a) bilinear interpolation, and (b) linear interpolation. 


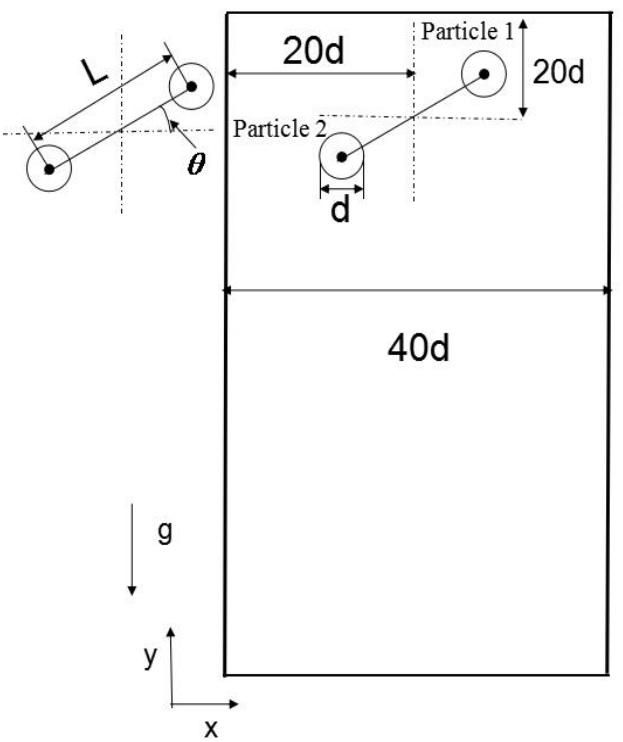

Fig. 2 Simulation schematic 


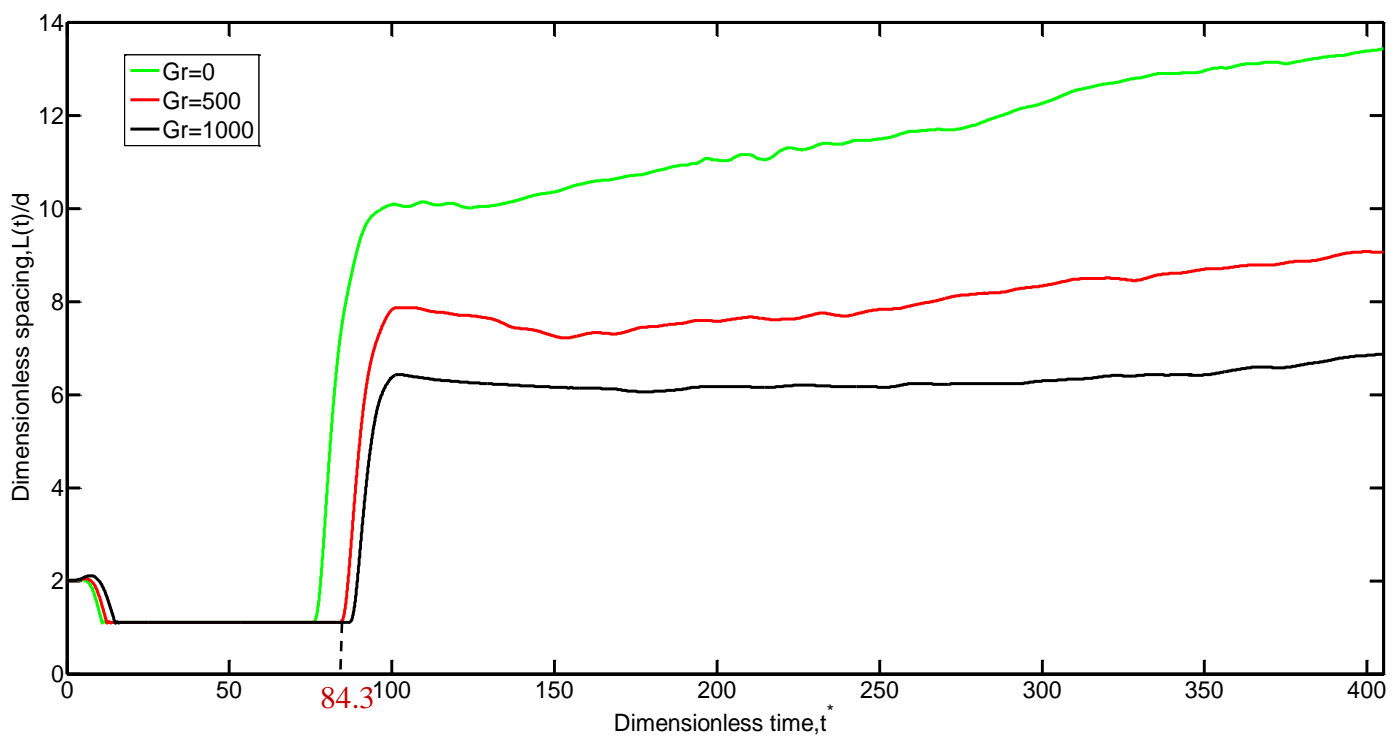

Fig. 3 Comparisons of dimensionless spacing of two hot particles at various Gr. 

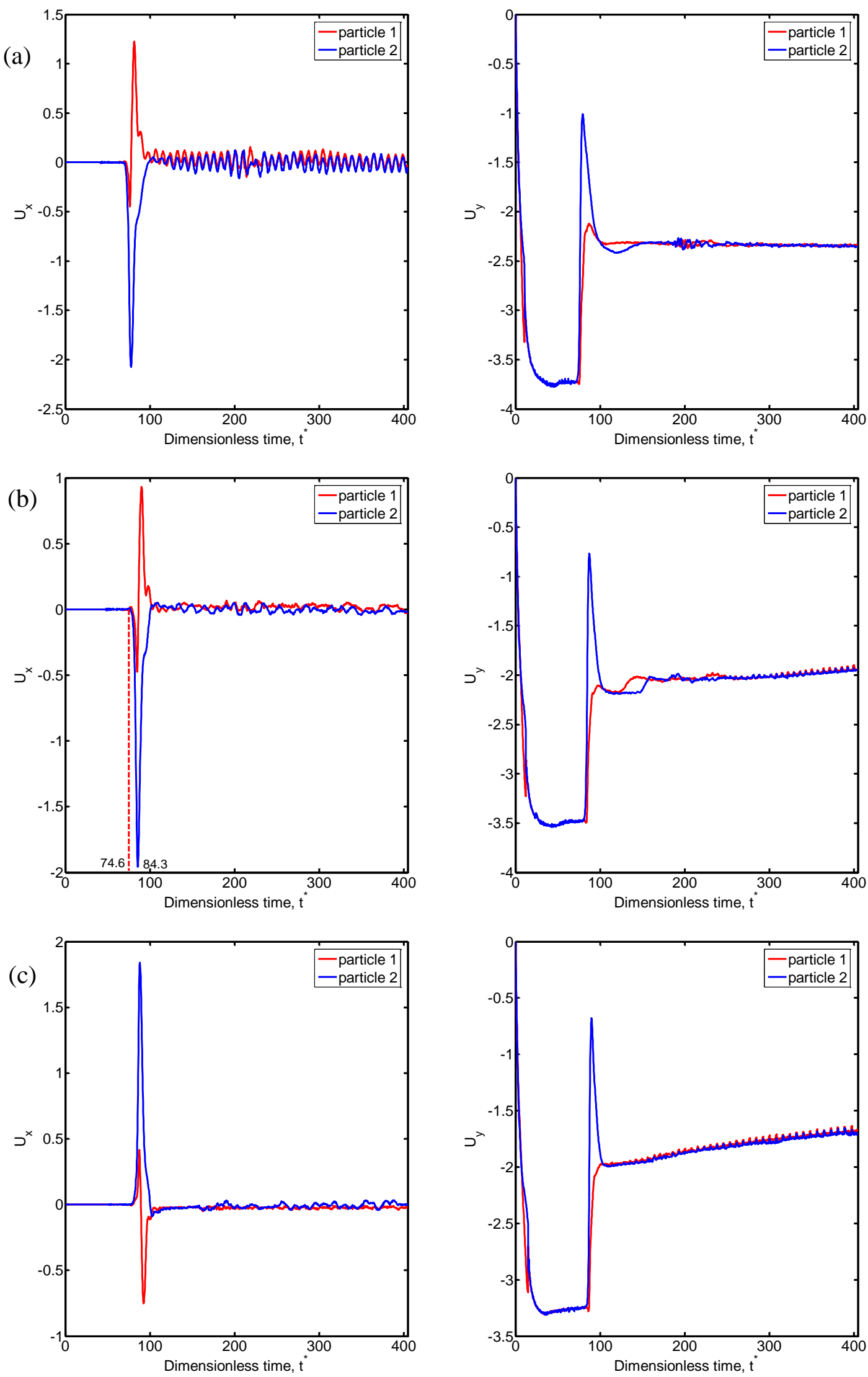

Fig. 4 Time evolution of horizontal velocity (left) and vertical velocity (right) of two hot particles at (a) $\mathrm{Gr}=0$; (b) $\mathrm{Gr}=500$ and (c) $\mathrm{Gr}=1000$. 
(a)

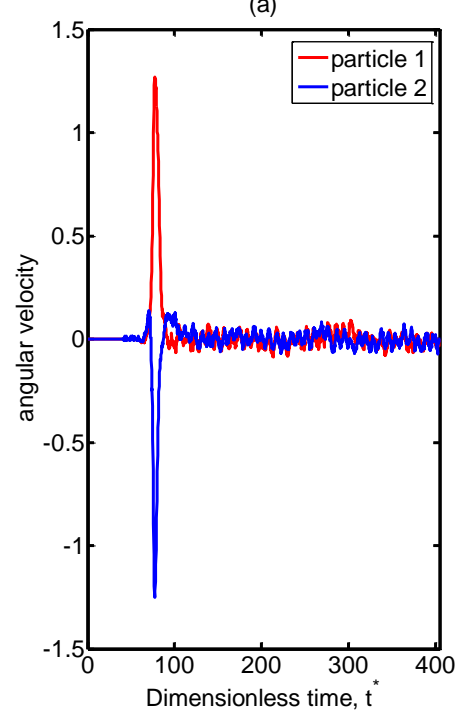

(b)

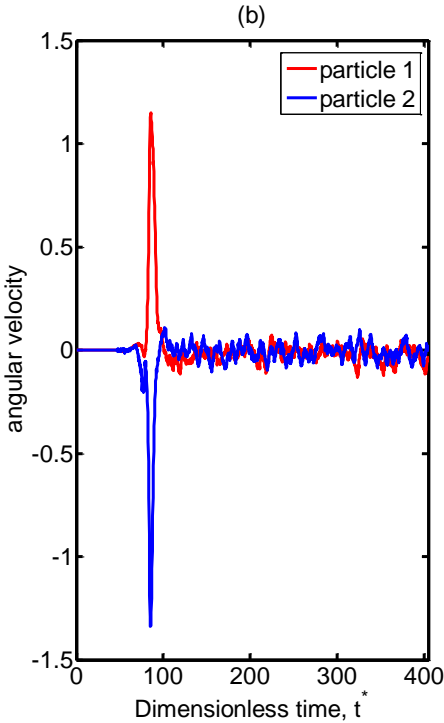

(c)

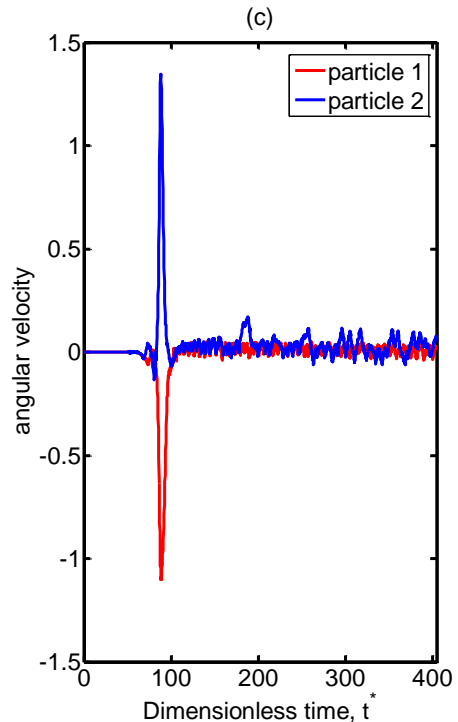

Fig. 5 Time evolution of angular velocities of two hot particles at (a) $\mathrm{Gr}=0$; (b) $\mathrm{Gr}=500$ and $\mathrm{Gr}=1000$. 


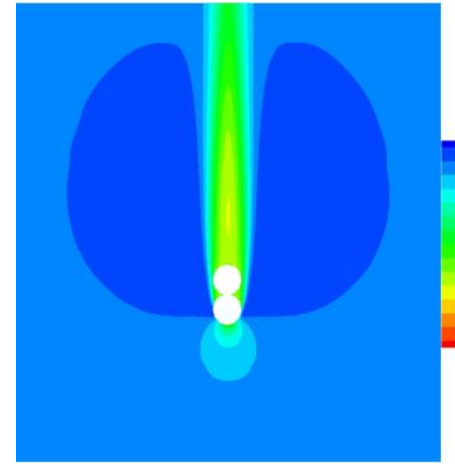

(a)

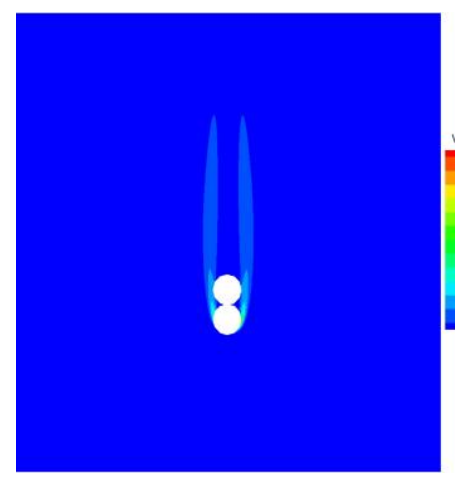

(b)

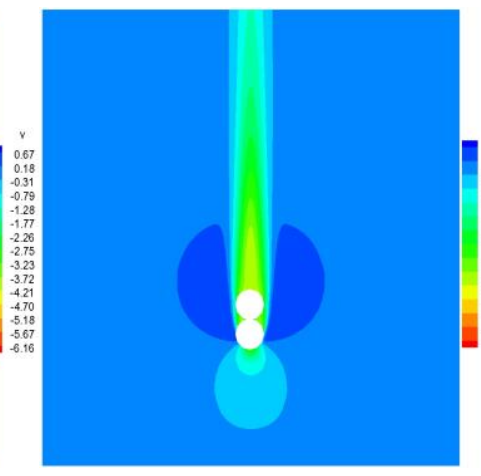

(c)

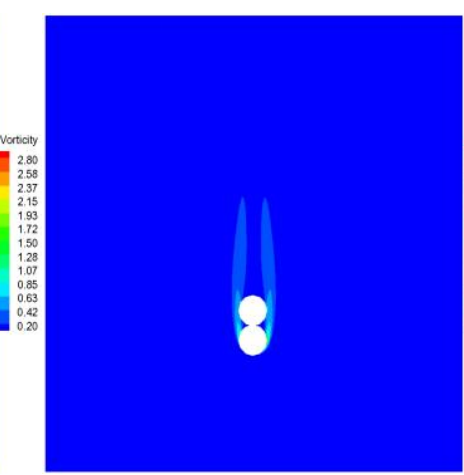

(d)

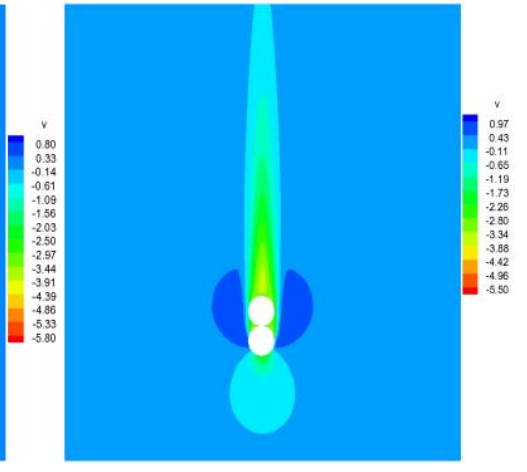

(e)

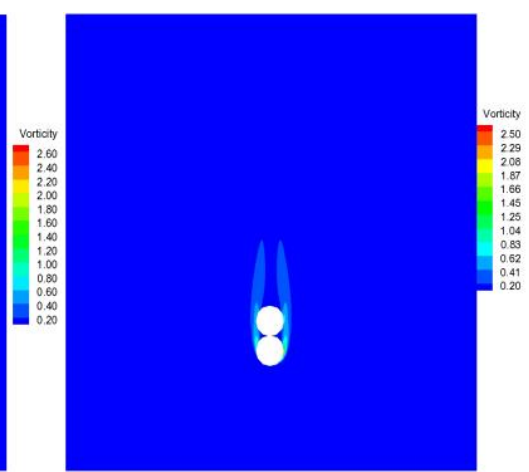

(f)

Fig. 6 Sedimentation of two hot particles at various $\mathrm{Gr}$ at $\mathrm{t}^{*}=57.85$. (a, b), (c, d,) and (e, f, ) are allocated for the cases of $\mathrm{Gr}=0,500,1000$ respectively. (a, c, e) vertical velocity contours, (b, d, f) vorticity magnitude contours. 

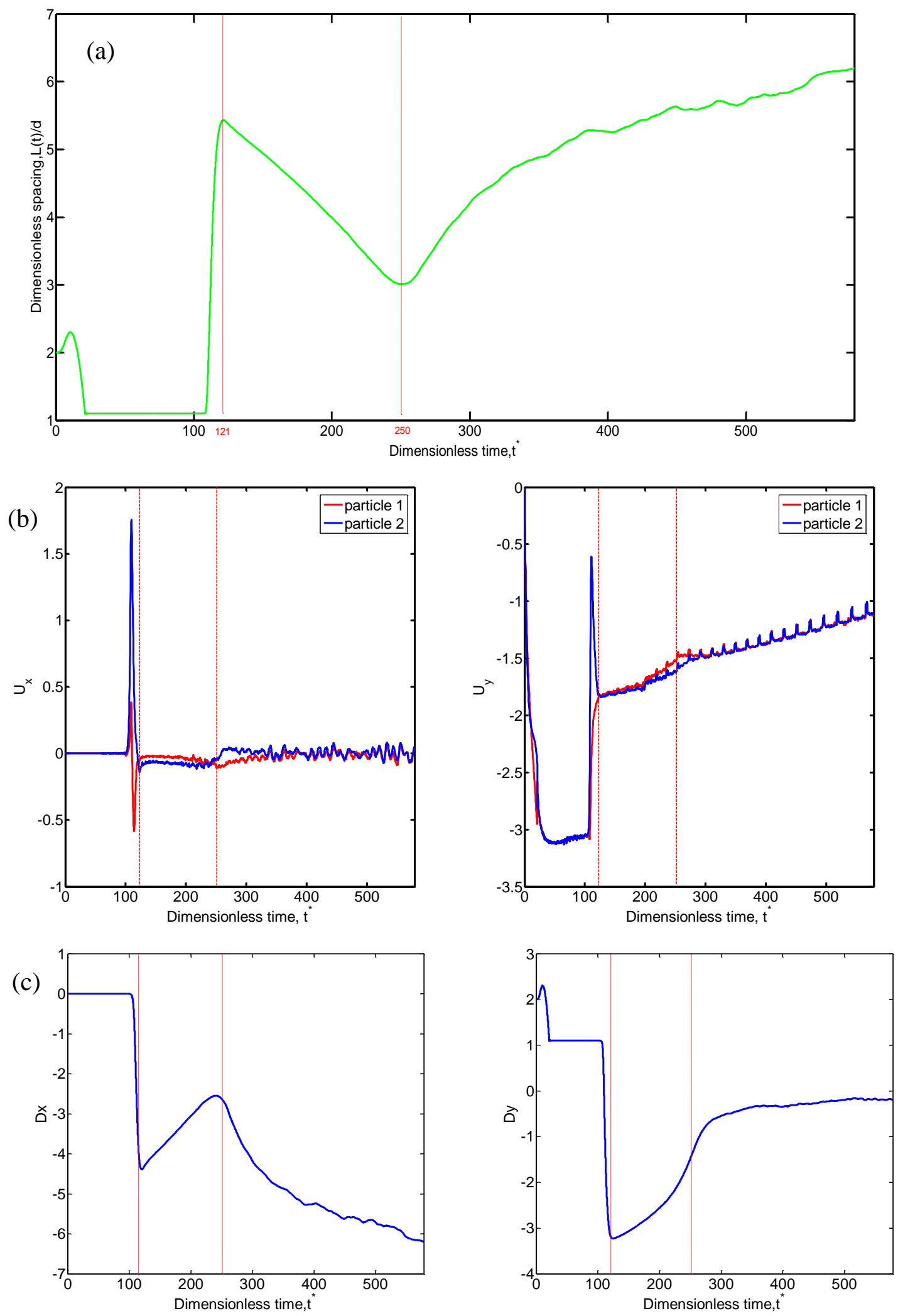

Fig. 7 Time evolution of (a) dimensionless distance, (b) settling velocity, and (c) distance component of two hot particles at $\mathrm{Gr}=1500$. The two red dotted lines indicate the time period within which another drafting process happens. Note that in (c) Dx $>0$, Dy $>0$ indicate the trailing particle locates on the right, upward side of the leading particle respectively. 

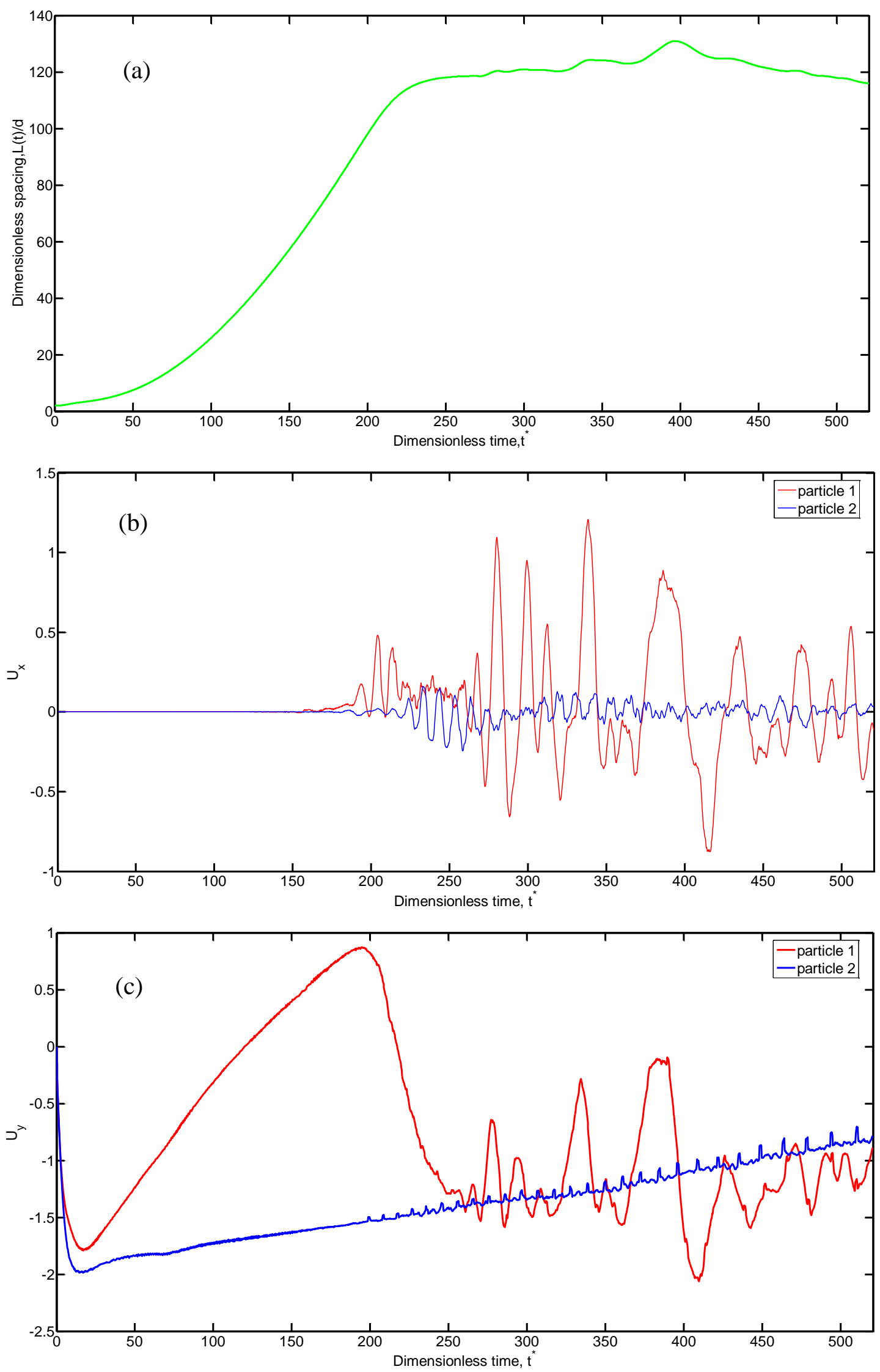

Fig. 8 Time evolution of (a) dimensionless distance, (b) horizontal velocity, and (c) vertical velocity of two hot particles at $\mathrm{Gr}=2000$. 


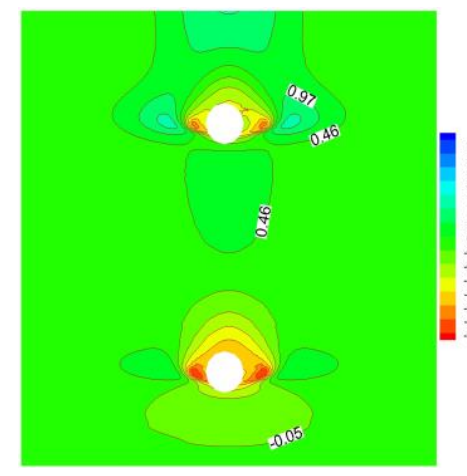

(a)

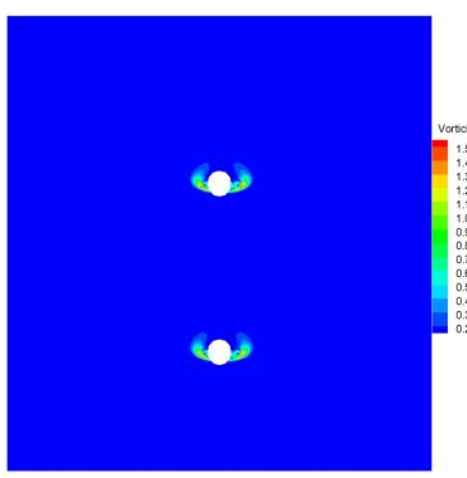

(b)

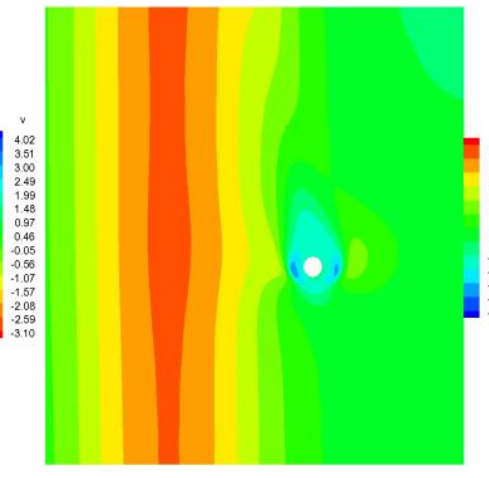

(c)

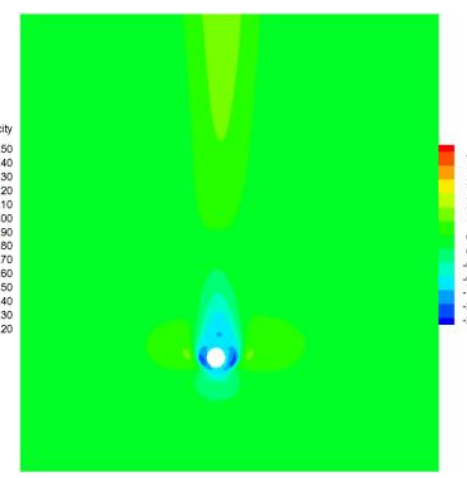

(d)

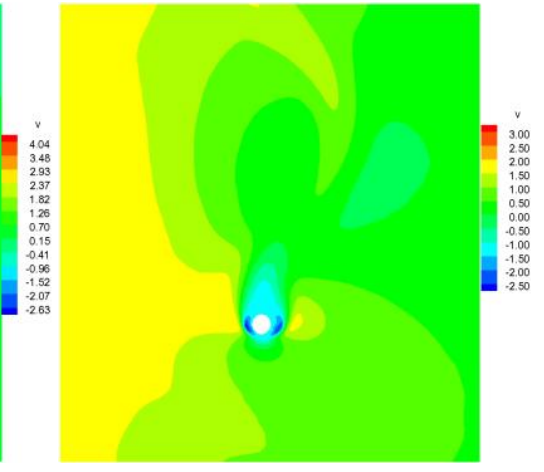

(e)

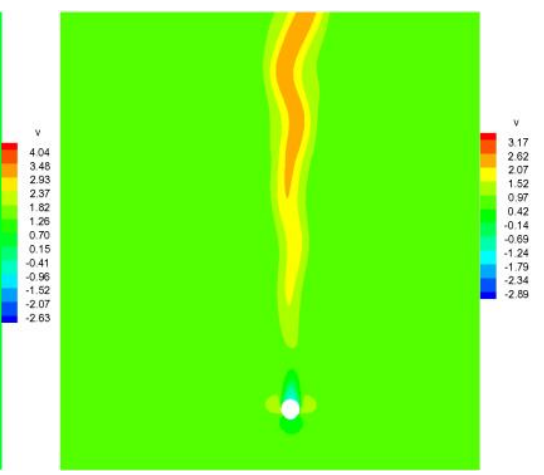

(f)

Fig. 9 Vertical velocity, vorticity magnitude contours of fluid field around the two hot particles at $\mathrm{Gr}=2000$. (a, b) at $\mathrm{t}^{*}=57.85,(\mathrm{c}, \mathrm{d})$ at $\mathrm{t}^{*}=231.4,(\mathrm{e}, \mathrm{f})$ at $\mathrm{t}^{*}=289.25$. Particularly, (c, e), (d, f) are the figures of local velocity region around the trailing particle and the leading particle respectively. 

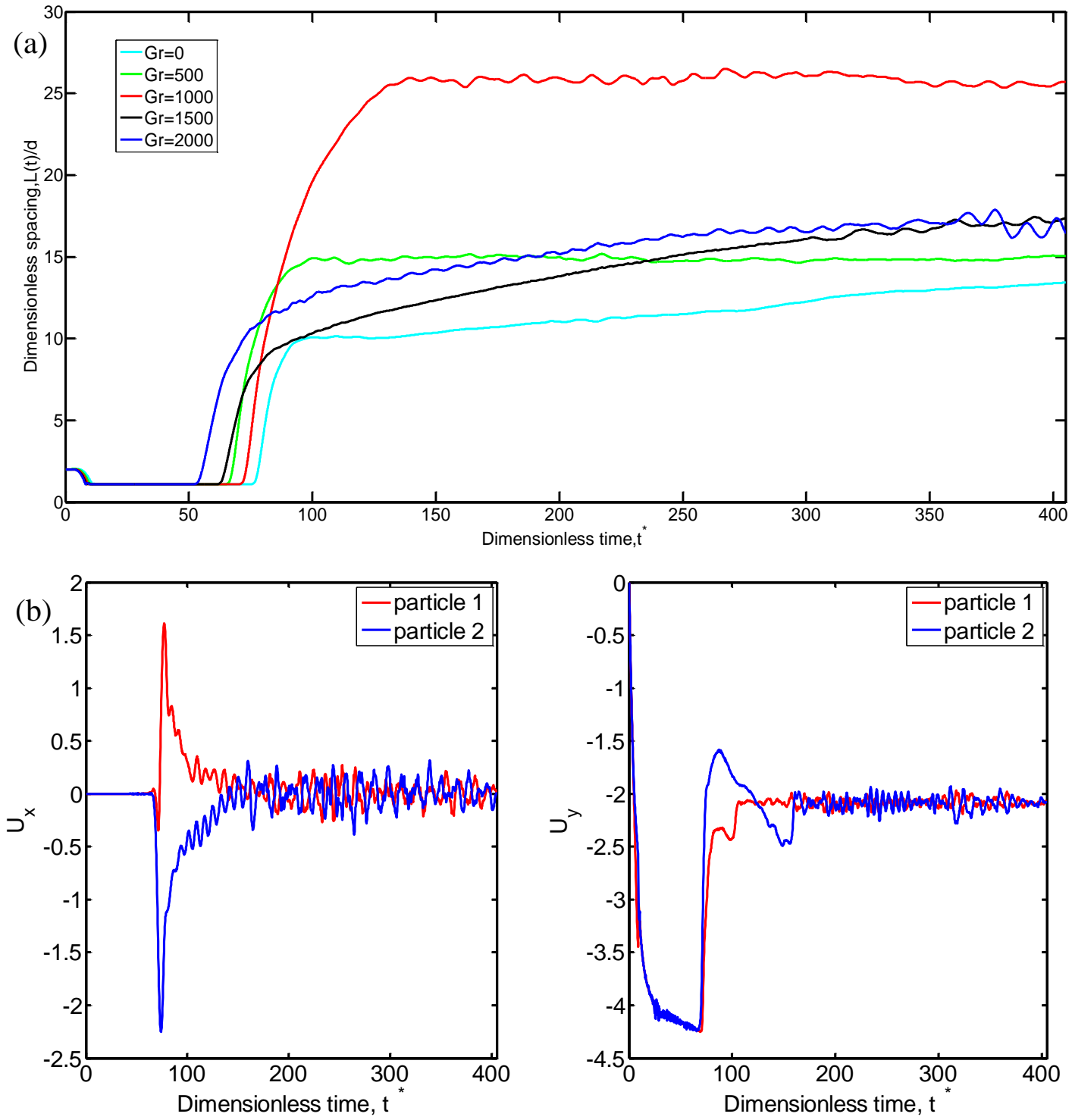

(c)
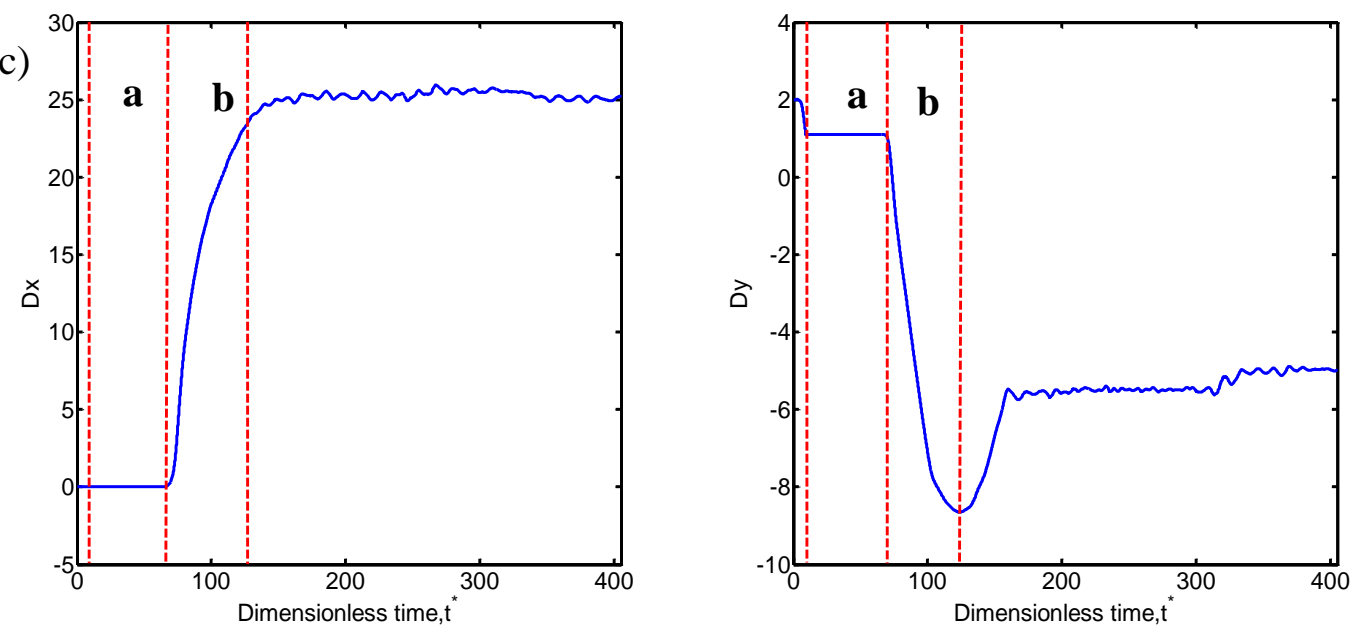

Fig. 10 Sedimentation of two cold particles. (a) Comparisons of dimensionless spacing of two cold particles at various Gr. (b) settling velocity, (c) horizontal and vertical distance of the two cold particles at $\mathrm{Gr}=1000$ ( $\mathrm{a}, \mathrm{b}$ indicate the tumbling, separating stage respectively). 


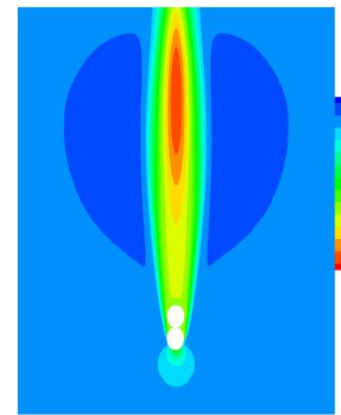

(a)

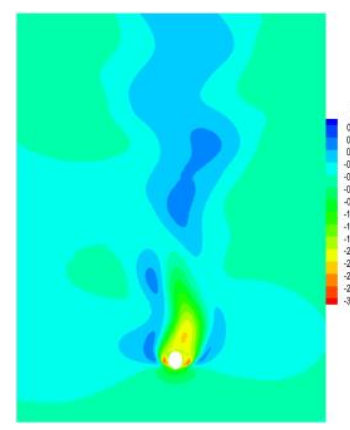

(e)

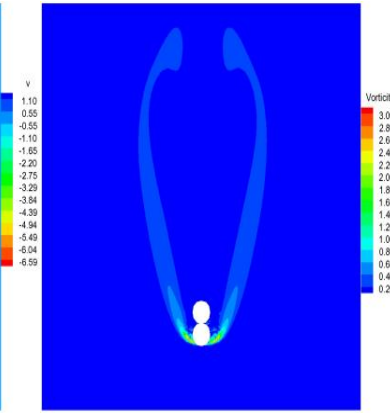

(b)

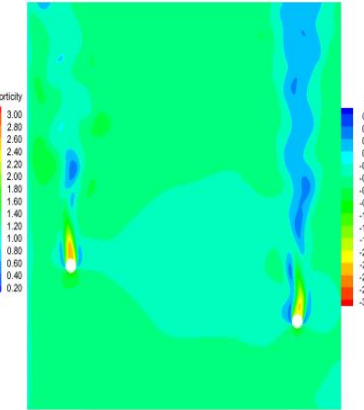

(c)

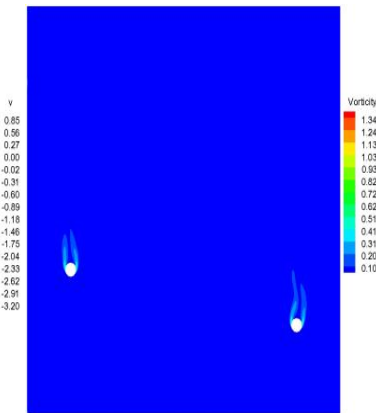

(d)

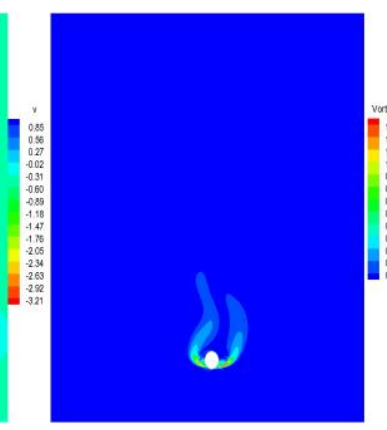

(f)

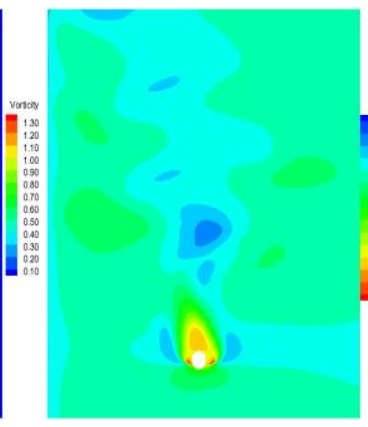

(g)

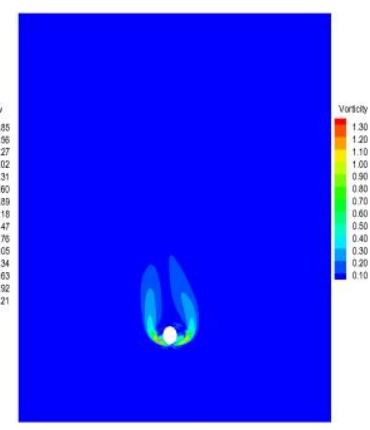

(h)

Fig. 11 Distributions of $(a, c)$ vertical velocity and $(b, d)$ vorticity magnitude of fluid field at $\mathrm{Gr}=1000$. $(\mathrm{a}, \mathrm{b})$ at $\mathrm{t}^{*}=57.85,(\mathrm{c}, \mathrm{d})$ at $\mathrm{t}^{*}=231.4$. Note that $(\mathrm{e}, \mathrm{f})$ and $(\mathrm{g}, \mathrm{h})$ are the magnifying figures of vertical velocity and vorticity magnitude around the trailing and the leading particle respectively at $t^{*}=231.4$. 

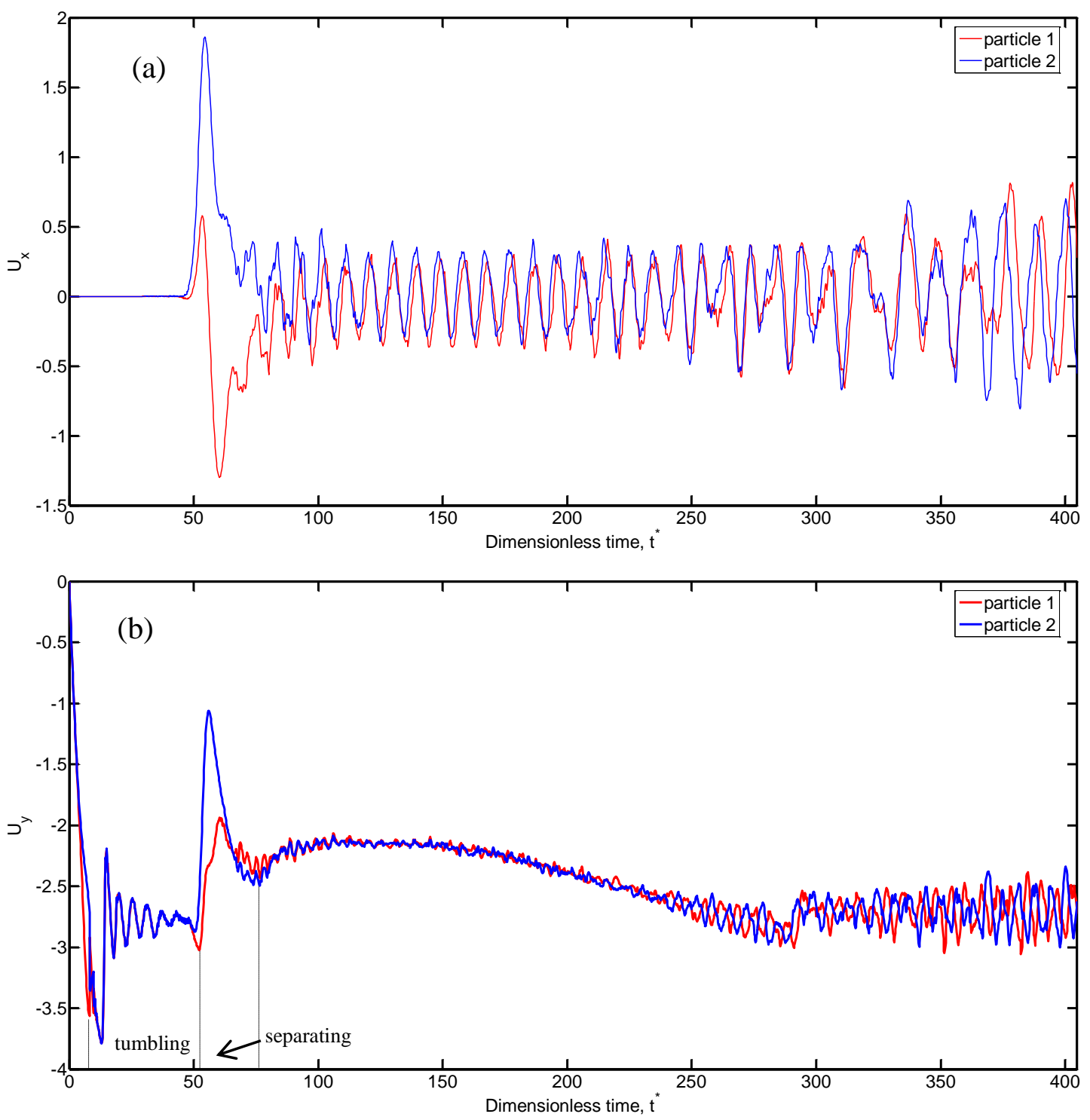

Fig. 12 Sedimentation of two cold particles at $\mathrm{Gr}=2000$. (a) horizontal and (b) vertical velocities of two cold particles as a function of time. 


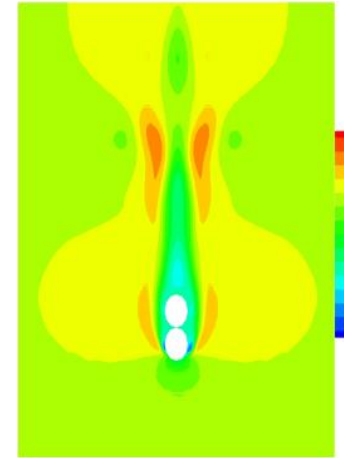

(a)

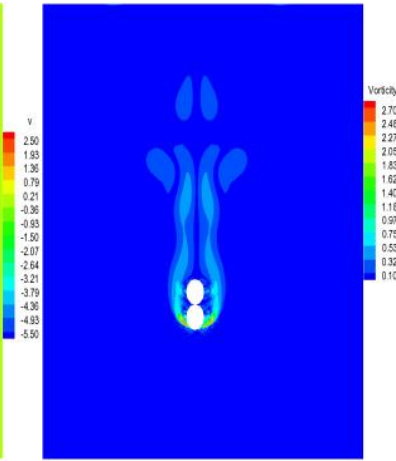

(b)

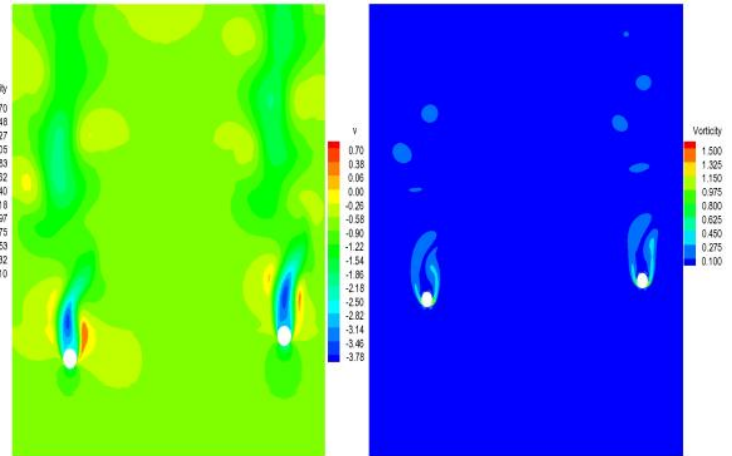

(c)

(d)

Fig. 13 Distributions of $(a, c)$ vertical velocity, (b, d) vorticity magnitude of fluid field at $\mathrm{Gr}=2000$. $(\mathrm{a}, \mathrm{b})$ at $\mathrm{t}^{*}=34.71,(\mathrm{c}, \mathrm{d})$ at $\mathrm{t}^{*}=208.26$. 


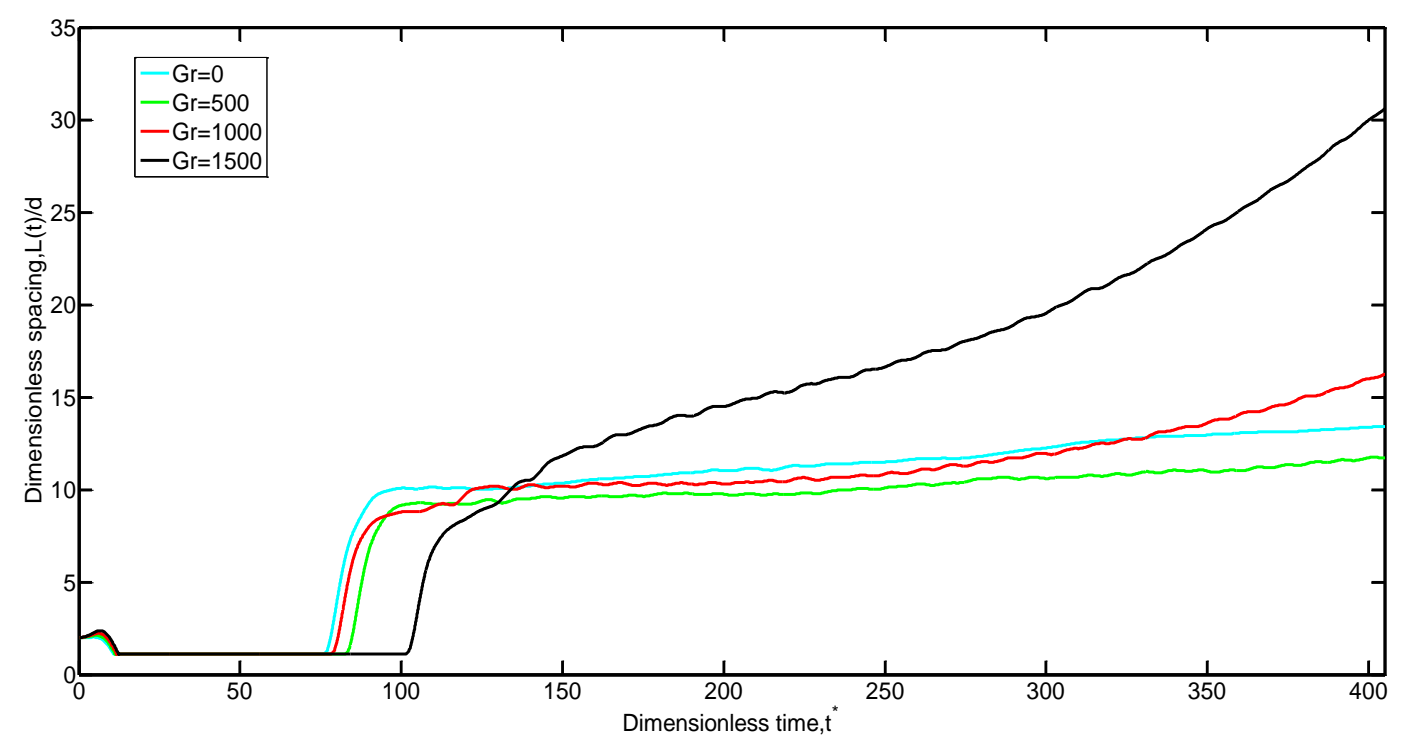

Fig. 14 Comparisons of dimensionless spacing of one hot and cold particle at various Gr. 

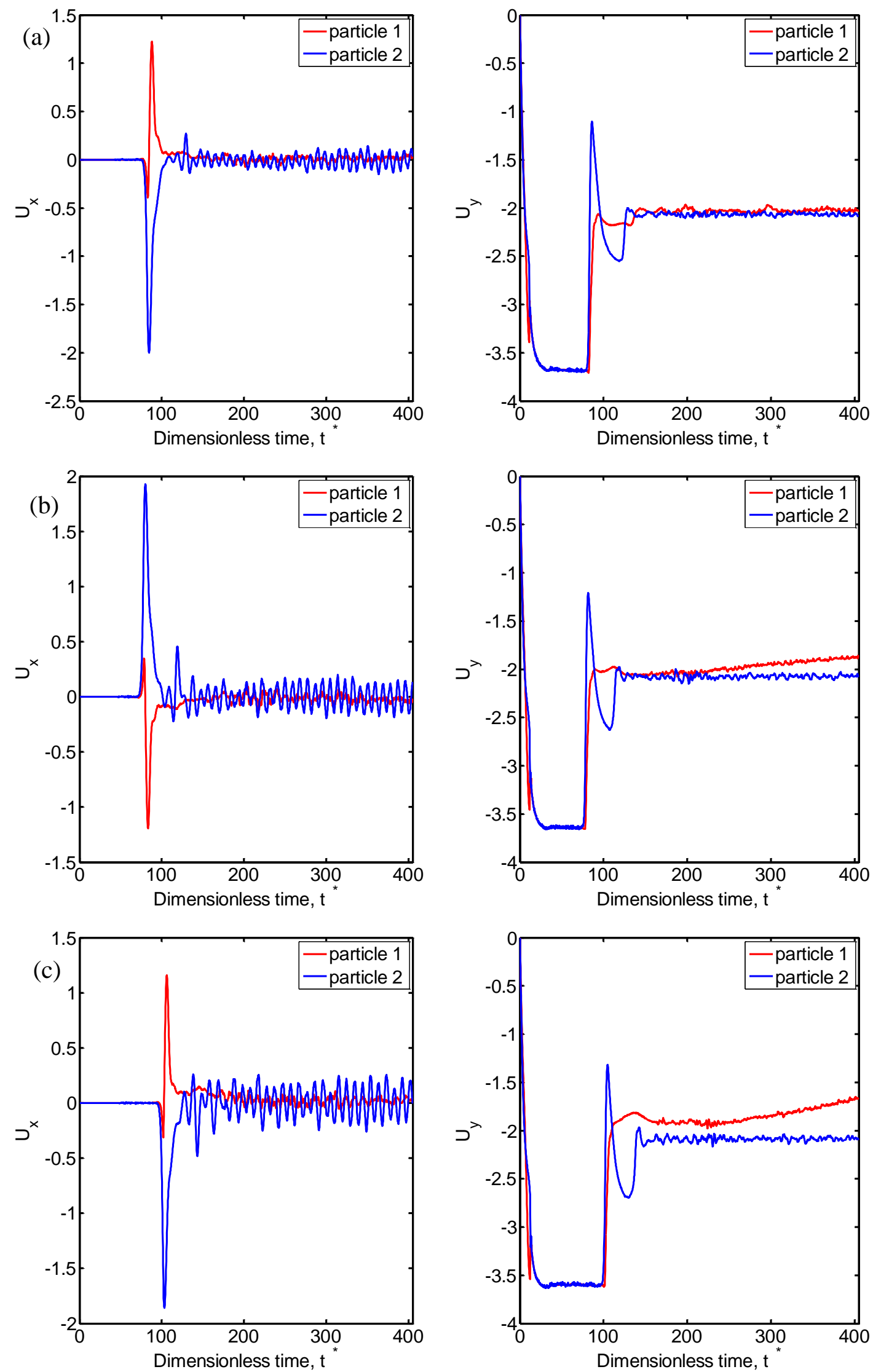

Fig. 15 Time evolution of horizontal velocity (left) and vertical velocity (right) of one hot and cold particle at (a) $\mathrm{Gr}=500$; (b) $\mathrm{Gr}=1000$ and (c) $\mathrm{Gr}=1500$. 

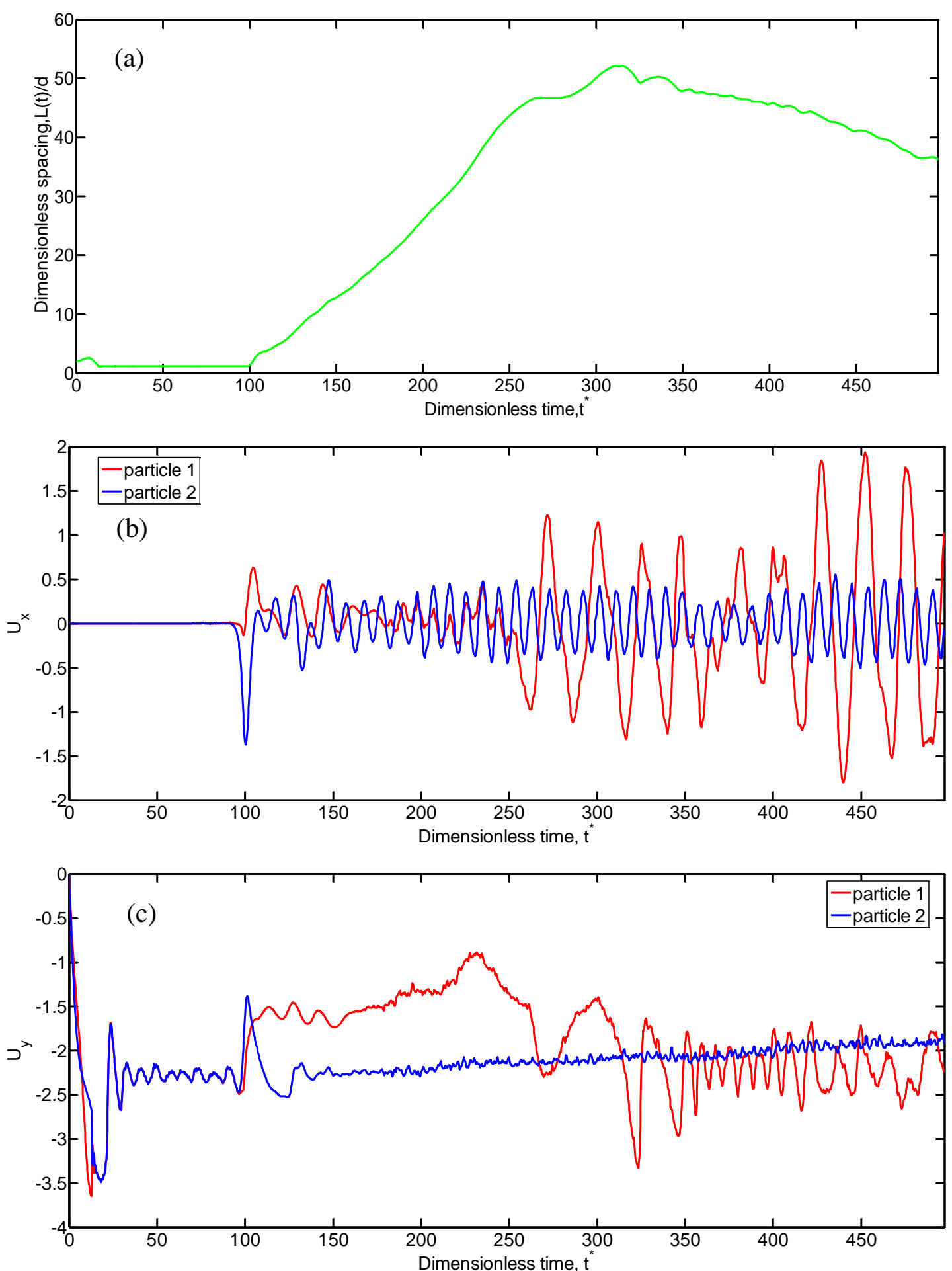

Fig. 16 Time evolution of (a) dimensionless distance, (b) horizontal velocity, and (c) vertical velocity of one hot particle and one cold particle at $\mathrm{Gr}=2000$. 


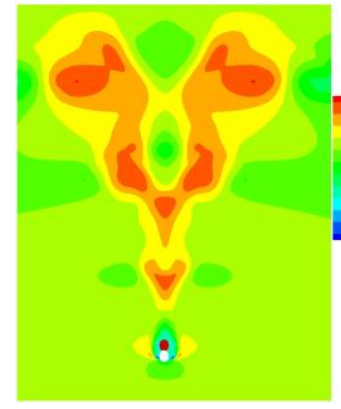

(a)

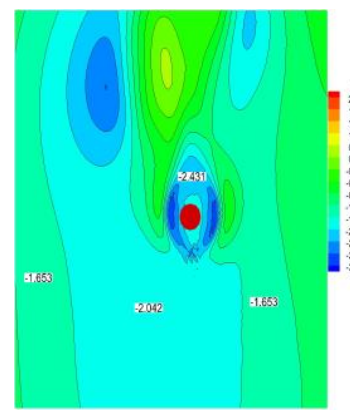

(e-1)

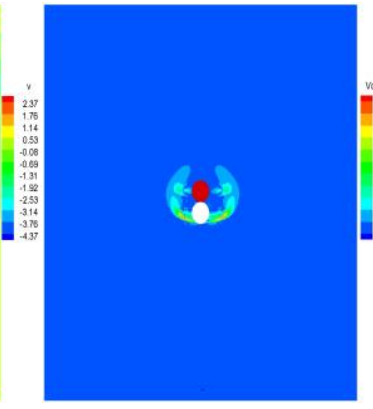

(b)

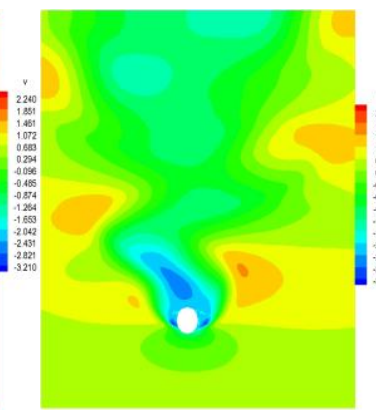

$(\mathrm{e}-2)$

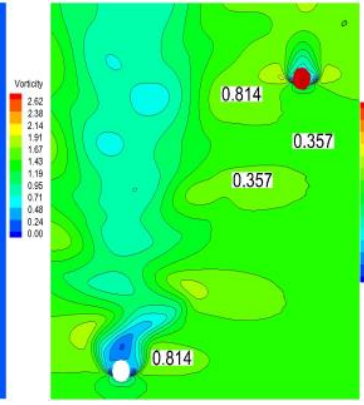

(c)

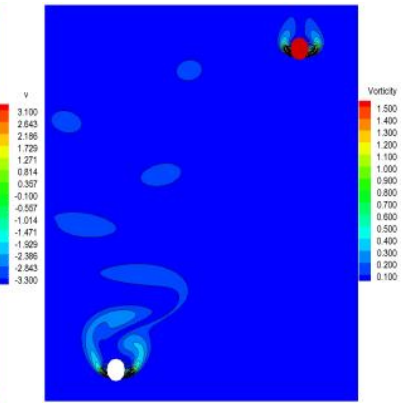

(d)

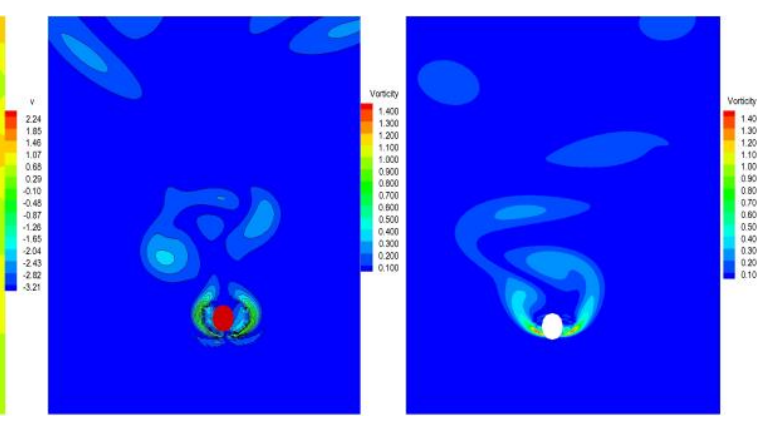

(f-1)

Fig. 17 Distributions of (a, c, e) vertical velocity and (b, d, f) vorticity magnitude of fluid field at $\mathrm{Gr}=2000$ at $\mathrm{t}^{*}=46.28,185.12,370.24$ respectively. Since flow field is much large, we take the regions around the two particles to study. Here, red and white circles indicate the hot and cold particle respectively. 

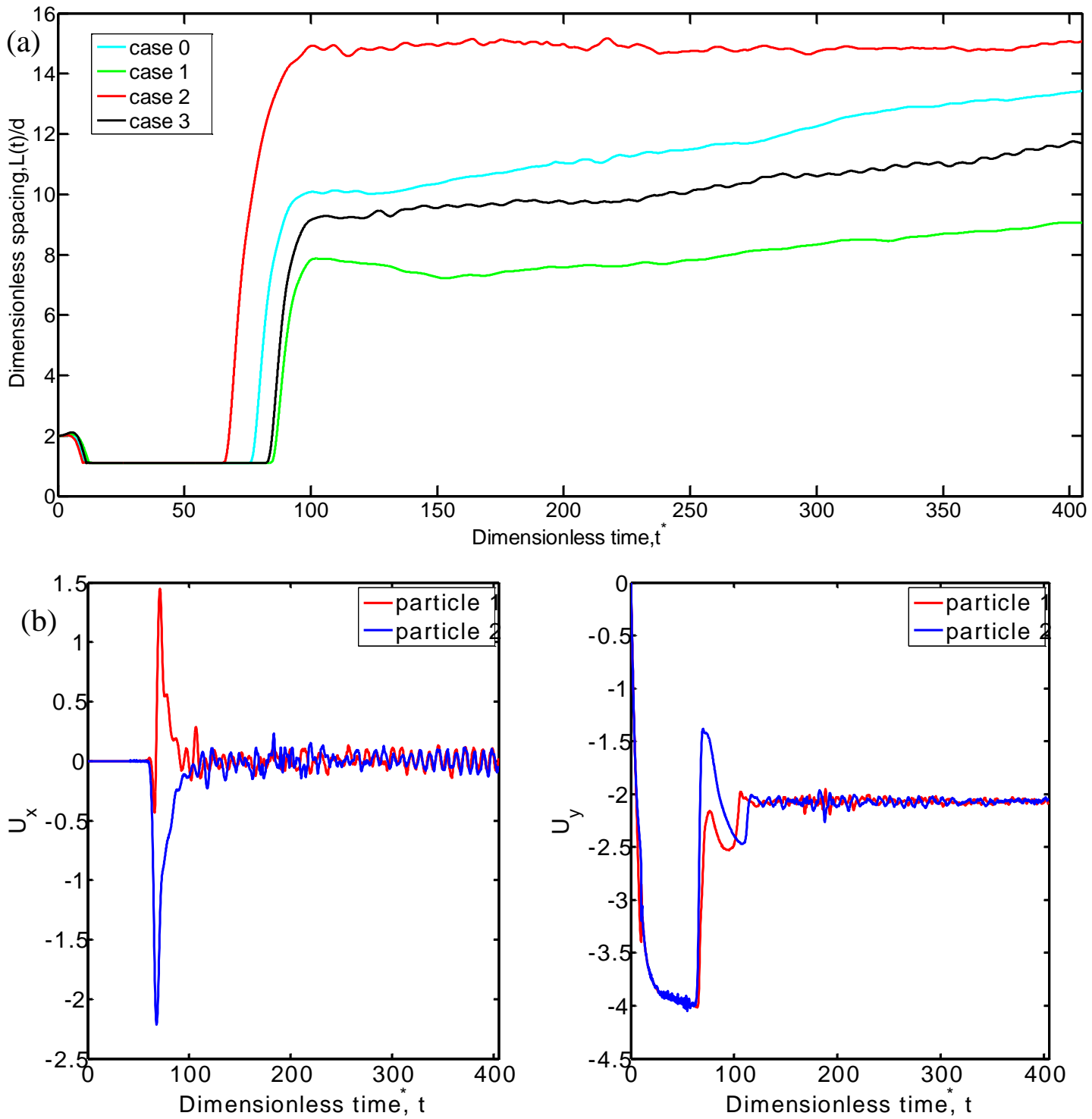

Fig. 18 (a) Comparison of two settling particles in the four cases at $\mathrm{Gr}=500$. (b) Time evolution of horizontal velocity and vertical velocity of two cold particles at $\mathrm{Gr}=500$. 


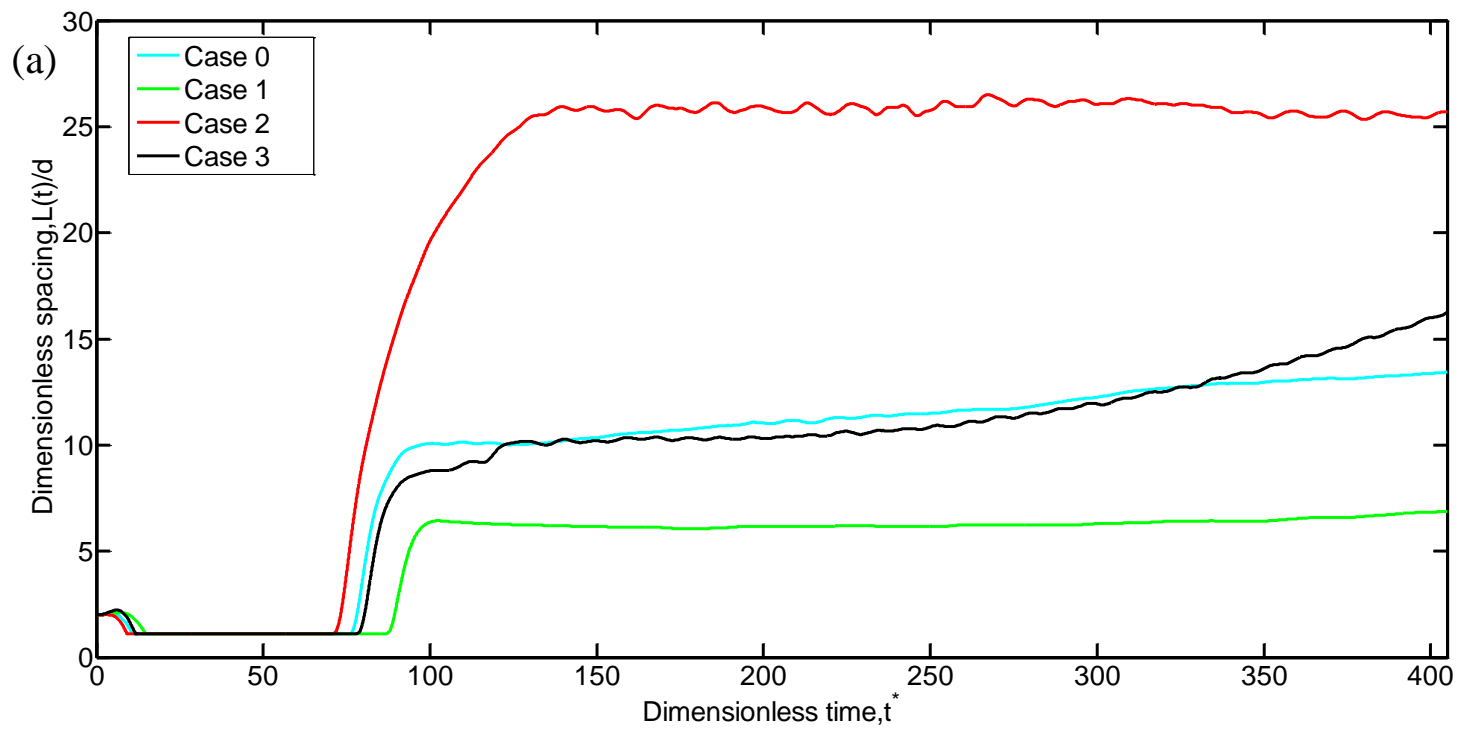

(b)

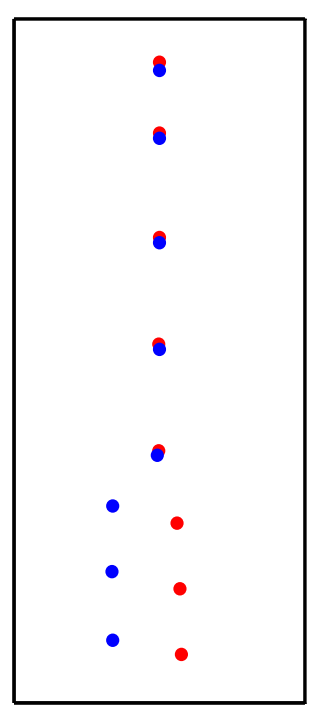

case 0

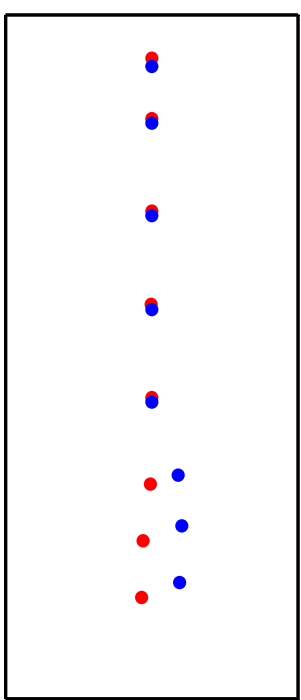

case 1

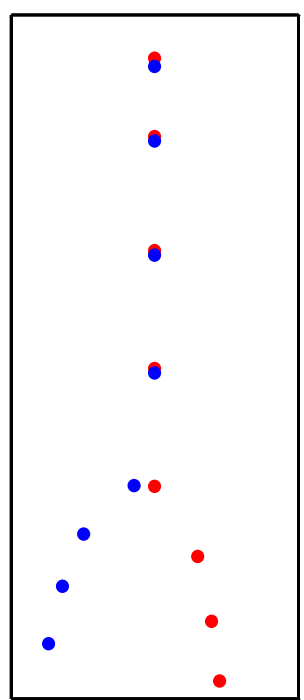

case 2

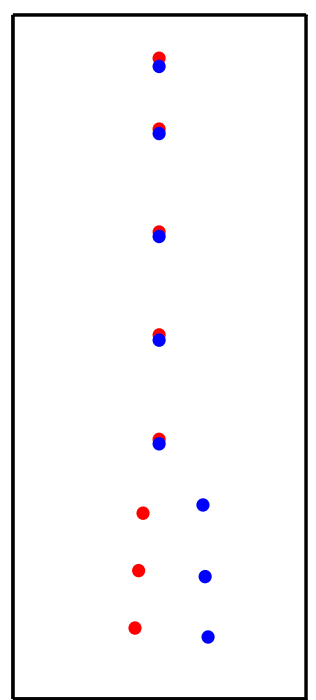

case 3

Fig. 19 Comparison of (a) dimensionless spacing and (b) trajectories of two settling particles in the four cases at $\mathrm{Gr}=1000$. 


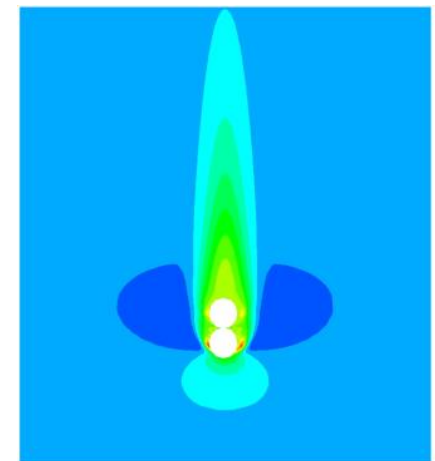

(a)

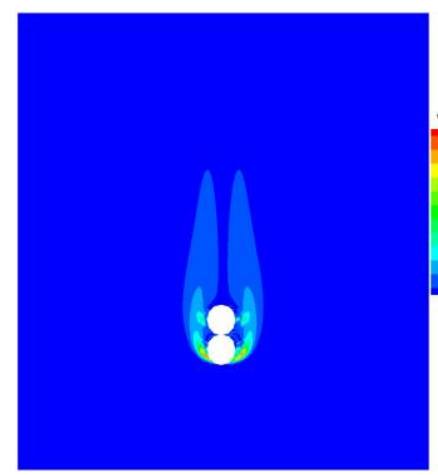

(d)

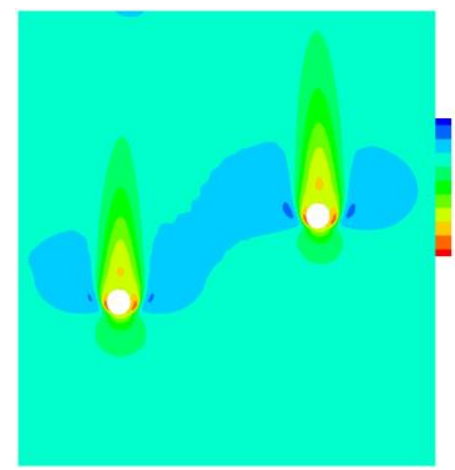

$(\mathrm{g})$

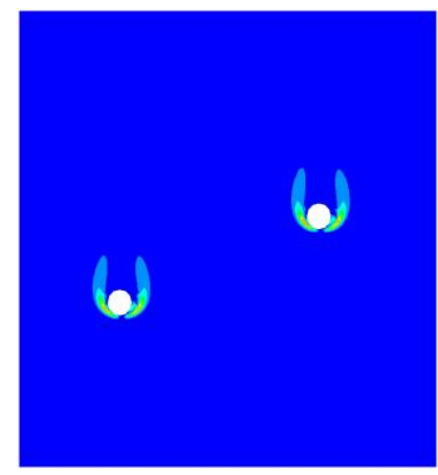

(j)

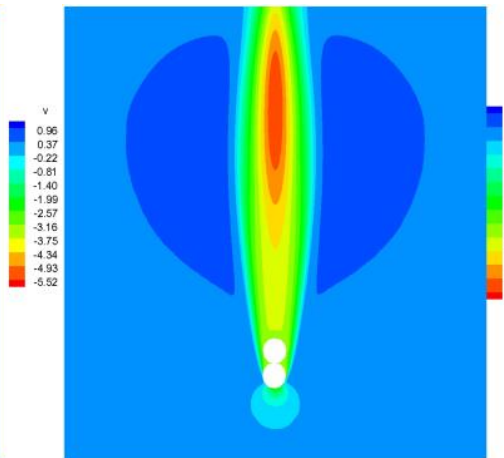

(b)

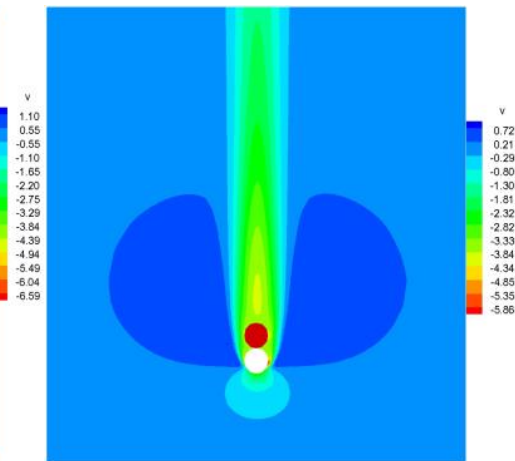

(c)

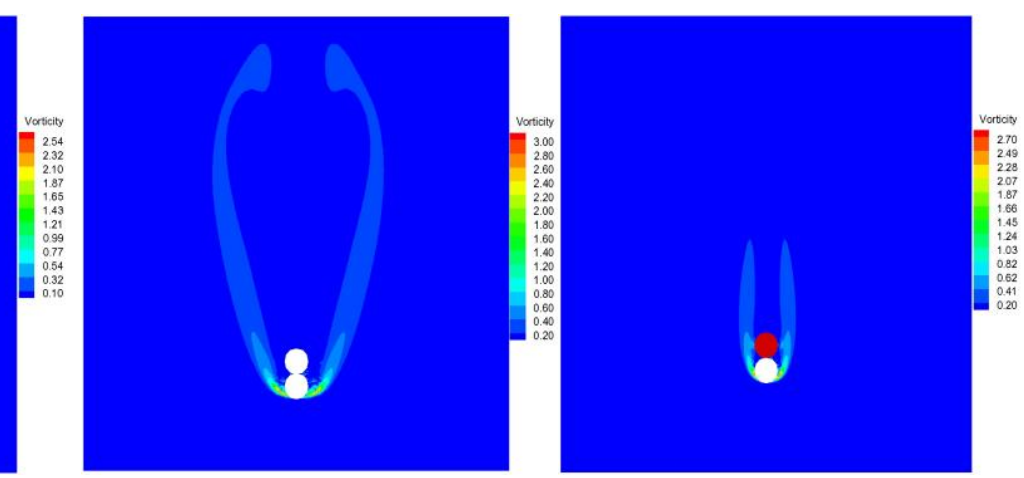

(f)

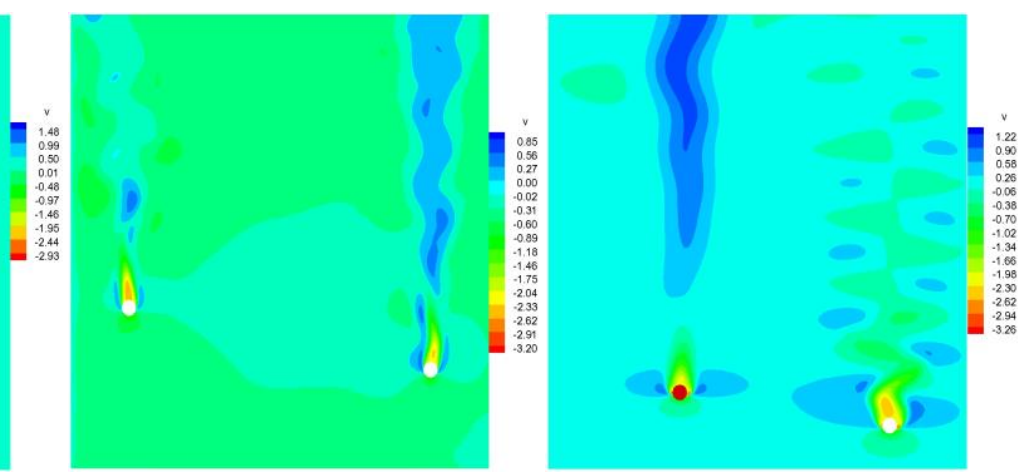

(h)

(i)

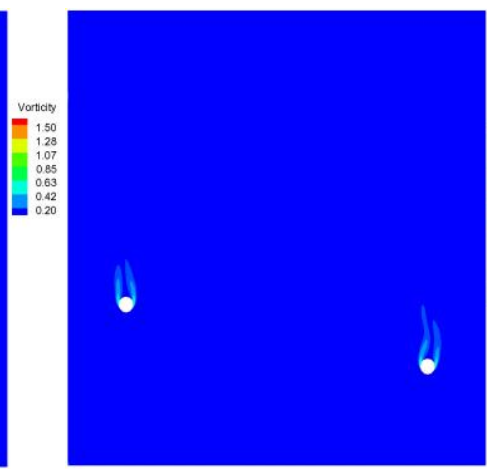

(k)

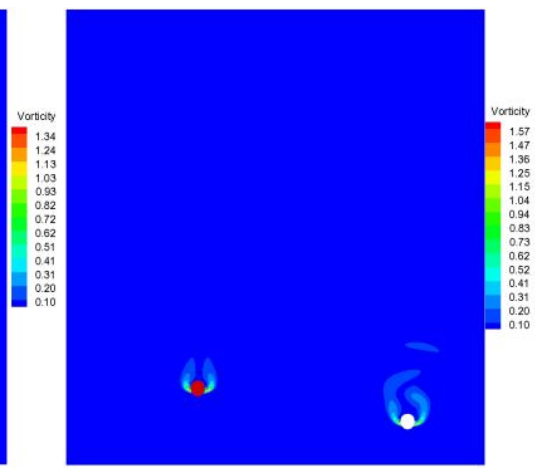

(m)

Fig. 20 Vertical velocity, and vorticity magnitude contours of fluid field: (a, d, g, j) for case 1, (b, $\mathrm{e}, \mathrm{h}, \mathrm{k}$ ) for case 2 , and $(\mathrm{c}, \mathrm{f}, \mathrm{i}, \mathrm{m})$ for case 3. Note that $(\mathrm{a}-\mathrm{f})$ for $\mathrm{t}^{*}=57.85$ and $(\mathrm{g}-\mathrm{m})$ for $\mathrm{t}^{*}=231.4$. 